Ref, TH . 1.659-CERN

\title{
CURRENT QUARKS, CONSTITUENT QUARKS, AND
}

SYMIETRIES OF RESONANCE DECAYS

\author{
A.J.G. Hey $\left.{ }^{*}\right)$, J.I. Rosner ${ }^{+}$) and J. Weyers \\ CERN - Geneva
}

\section{A B S T R A C T}

The transformation between "current" quarks and "constituent" quarks.recently suggested by Melosh is exainined with respect to its predictions for pionic decays of resonances. It implies the use of $\mathrm{SU}(6)_{W}$ for classifying particle states but not for describing decay processes. Instead, pion emission proceeds via $\Delta I_{z}=0, \pm 1$, where. I is the internal ("quark") orbital angular momentum. This decay symmetry is called $\mathrm{SU}(6)_{\mathrm{W}}\left[\Delta \mathrm{L}_{z}=0, \pm 1\right]$. It is proven equivalent for any decay $A \rightarrow B+\pi$. (where $A, B$ are arbitrary $q \bar{q}$ or $q q q$ hadrons) to the ${ }^{3} P_{0}$ quark-pair creation model for such decays, as formulated by Micu, Colglazier, Petersen and Rosner. The roles of final orbital angular momenta $l$ and of $S U(3) \times S U(3)$ subgroups of $S U(6)_{W}$ are also discussed, and some new predictions are made for decays of meson resonances below $1700 \mathrm{MeV}$.

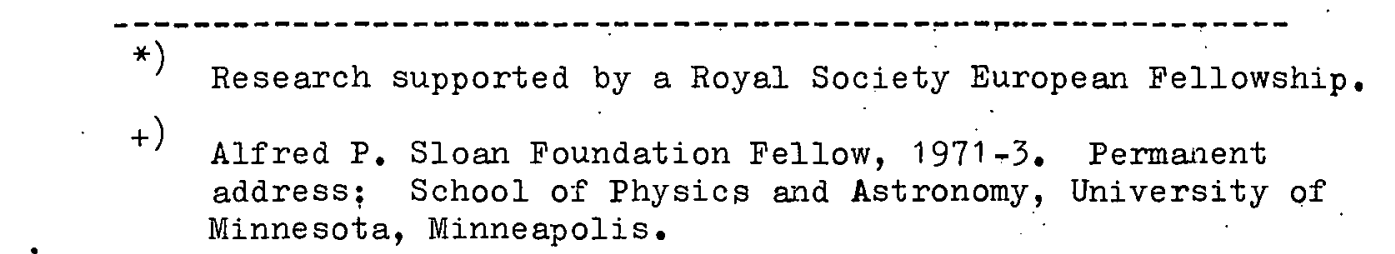

* Research supported by a Royal Society European Fellowship.

) Alfred P. Sloan Foundation Fellow, 1971-3. Permanent Minnesota, Minneapolis.

Ref.TH. 1659-CERH

18 April 1973

This report wos $N$ OICE

This report was prepared as an account of work sponsored by the United States Government. Neither Commission, States nor the United States Atomic Energy Commission, nor any of their employees, nor any of their contractors, subcontractors, or the ir employees, makes any warranty, express or implied, or assumes any legal liability or responsibility for the accuracy, completeness or usefulness of any information, apparatus, product or process disclosed, or represents that its use would not infringe privately owned rights. 


\section{DISCLAIMER}

This report was prepared as an account of work sponsored by an agency of the United States Government. Neither the United States Government nor any agency Thereof, nor any of their employees, makes any warranty, express or implied, or assumes any legal liability or responsibility for the accuracy, completeness, or usefulness of any information, apparatus, product, or process disclosed, or represents that its use would not infringe privately owned rights. Reference herein to any specific commercial product, process, or service by trade name, trademark, manufacturer, or otherwise does not necessarily constitute or imply its endorsement, recommendation, or favoring by the United States Government or any agency thereof. The views and opinions of authors expressed herein do not necessarily state or reflect those of the United States Government or any agency thereof. 


\section{DISCLAIMER}

Portions of this document may be illegible in electronic image products. Images are produced from the best available original document. 
The quark model has met with remarkable success in hadronic physics, both as a "coristituent" model for hadrons 1) and as a model for their weak and electromagnetic currents 2),3)。

The two quark pictures are associated with two different $\mathrm{SU}(6)$. algebras of operators. The algebra SU(6) $\mathrm{W}$, strong introduced by Lipkin and Meshkov 4) seems appropriate for the classification of the lowest lying hadrons: they appear to fall into simple irreducible representations of this symmetry. Thus, this "constituent" picture places the observed mesons in 1 and 35. dimensional representations and the observed baryons in 56 and 70 dimensional representations of $\mathrm{SU}(6)_{\mathrm{W}, \text { strong }}$.

A different $S U(6)$ algebra introduced by Dashen and Gell-Mann ${ }^{5)}$, which one may call $S U(6)_{W}$, currents' is obeyed by the socalled "good" charges in the infinite momentum limit ${ }^{6)}$. These charges are the integrals over local densities which are directly or indirectly measurable in weak and electromagnetic processes. Moreover, since the isovector axial charge is included in this algebra, and pionic decays of resonances $A \rightarrow B+\pi$ are related by $P C A C$ to the matrix element of this axial charge, one expects that $S U(6)_{W}$, currents may provide a description of some purely hadronic processes as well.

Conceptually, constituent quarks and current quarks, and hence their respective $\mathrm{SU}(6)_{\mathrm{W}}$ algebras, are not necessarily identical. The success of CVC means that the SU(3) subalgebras are identical, but an attempt to identify particle states with pure representations of $\mathrm{SU}(6)_{\mathrm{W}}$, currents leads to a number of difficulties. Notable among these are the prediction that Adler-Weisberger relations 7) should be well-saturated with the lowest $\frac{1}{2}^{+}$and $\frac{3}{2}^{+}$baryon resonances (which they are not 7 ), 8) ), and that anomalous magnetic moments of baryons should vanish (which they do not) 5). Moreover, although a protion is well-classified as a state of three "constituent" quarks, it looks considerably more complicated when probed with currents. In such a description (based on deep inelastic lepton scattering 9) the proton seems to contain additional quark-antiquark pairs and perhaps some neutral "glue" as well 3). Hence, although physicaily observable transitions are best described in the language of $\mathrm{SU}(6){ }_{\mathrm{W}}$, currents' the fact that the states themselves are complicated mixtures of representations of this group ${ }^{10)}$ has been a substantial obstacle up to now to concrete applications of this symmetry. 
Recently Melosh 11) has constructed the transformation between the two $\mathrm{SU}(6)_{\mathrm{W}}$ algebras in the free quark model. He finds that this transformation $V$, acting on the axial charge. $F^{i 5}$, produces a transformed axial charge $\widetilde{F}^{i 5} \equiv V^{-1} \mathrm{~F}^{i 5} \mathrm{~V}$ with relatively simple properties in the constituent quark picture.

Abstracting from this "free quark" property, several
authors 12),13) have assumed that a similarly simple $\widetilde{F}^{i 5}$ exists in the interacting theory as well. The matrix elements of $\mathrm{F}^{i 5}$ between pure

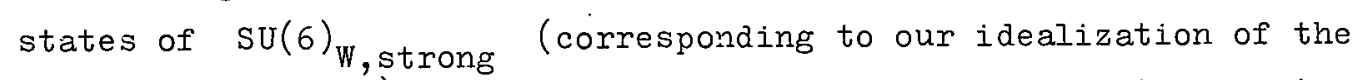
physical particles) are then relatively straightforward to compute, and imply a number of relations among pionic decays of resonances.

In this paper we examine the general structure of such

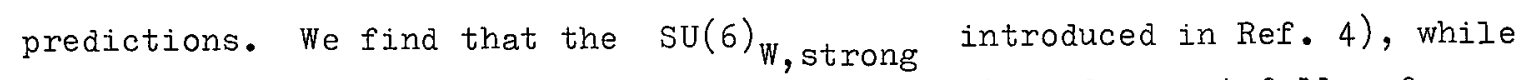
perhaps appropriate for classifying particle states, does not follow for collinear processes such as resonance decays. This "wrong" symmetry will be referred to as "nalve" $\mathrm{SU}(6)_{W}$. Instead, in the present scheme, some relatively simple selection rules govern pionic transitions between states classified according to $\mathrm{SU}(6)_{\mathrm{W}, \text { strong }}$. In terms of an abstractly defined internal orbital angular nomentum $\mathrm{L}_{z}$, these transitions turn out to involve only $\Delta I_{z}=0, \pm 1$. This "possibly right" decay symmetry will thus be referred to as $\mathrm{SU}(6)_{\mathrm{W}} \quad\left[\Delta \mathrm{I}_{\mathrm{Z}}=0, \pm 1\right]$.

We find that for any hadronic decay. $A \rightarrow B+\pi$, the symmetry $\mathrm{SU}(6)_{\mathrm{W}}\left[\overline{\Delta I}_{\mathrm{z}}=0, \pm 1\right]$, motivated by the work of Ref。11), is equivalent to the successful phenomenological " ${ }^{3}$ " model of Micu 14 , , Colglazier and Rosner ${ }^{15)}$ and Petersen and Rosner 16). The phenomenological prescription 14)-19) known as " $\ell$ broken $\left.\left.\mathrm{SU}(6)_{\mathrm{W}} " 16\right), 17\right)$ is found to be equivalent to $\left.\mathrm{SU}(6)_{\mathrm{W}} \quad \Delta_{\mathrm{z}}=0, \pm 1\right]$ for many cases of physical interest, but a special case of this symmetry (and hence essentially unmotivated on theoretical grounds) in general. We have also derived the predictions of the chiral $\mathrm{SU}(3) \times \mathrm{SU}(3)$ strong subalgebra of $\mathrm{SJ}(6)_{\mathrm{W}, \text { strong, and }}$ discuss their relation to other symmetries in some specific mesonic cases. Various phenomenological applications to decays of the mesons. in the "R" region (mass around $1700 \mathrm{MeV}$ ) are mentioned. 
The paper is organized as follows. Sections 1 and 2 are devoted, respectively, to the constituent and current quark approaches to $\mathrm{SU}(6)_{\mathrm{W}}$ as applied to hadronic vertices. These sections, as well as the beginning of Section 3 which deals with the explicit expressions for decay amplitudes, are expository in nature and follow closely the treatments of Refs. 11)-13). Then, in the remainder of section 3, we discuss in some detail all possible pionic decays of meson resonances up to $I=2$. This Section includes predictions of interest to experimentalists. Section 4 treats the equivalences and differences among the various above-mentioned pictures of hadronic decays. Finally, in Section 5, we summarize our results and indicate where further work is needed.

\section{CONSTITUENT QUARKS}

Particle states at rest appear to be well described by the quark model with orbital excitations. By taking all mesons as quarkantiquark pairs and all baryons as three quarks one can then understand a number of features of the hadron spectrum.

The symmetry associated with the above classification is $U(6) \times U(6) \times 0(3)$. The first $U(6)$ refers to quarks and the second to antiquarks, while the $O(3)$ describes the orbital angular momentum $I$. A number of multiplets of this group are either entirely filled or well on their way to being so. There is strong evidence for mesonic states belonging to $(\underset{\sim}{\sigma}, \bar{\sigma}), \mathrm{I}^{\mathrm{P}}=0^{-}, 1^{+}$and $2^{-}$and for the baryonic multiplets. $\left.\left.[56,1), I^{P}=0^{+}\right],[70,1), I^{P}=1^{-}\right]$and $\left[(56,1), I^{P}=2^{+}\right]$. Other mesonic and baryonic multiplets may exist roughly degenerate with the $\mathrm{I}=2$ levels just mentioned.

$$
\text { Clearly this } U(6) \times U(6) \times 0(3) \text { symmetry, which we shall }
$$
call the rest symmetry, has to be badly violated in resonance decays since it forbids, e.g०, $\Delta \rightarrow \mathbb{N} \pi$ or $p \rightarrow \pi \pi$. This is, of course, not surprising since decay amplitudes involve moving particles for which the rest symmetry should not hold. Lipkin and Meshkov ${ }^{20)}$ pointed out that the reflection and rotation invariance properties of collinear processes, such as twobody decays, lead.to invariance under "W spin" or $\mathrm{SU}(2)_{\mathrm{W}}$. For a particle moving along the $z$ axis, the generators of $W$ spin may be written 


$$
W_{x}=P_{\text {int }} S_{x} ; W_{y}=P_{\text {int }} S_{y} ; W_{z}=S_{z}
$$

where $P_{\text {int }}$ is the intrinsic parity of the system. For a single free quark moving in the $\hat{z}$ direction one would thus write the $S U(2)_{W}$ generators as follows

$$
W_{x}=\beta \frac{\sigma_{x}}{2} \quad ; W_{y}=\beta \frac{\sigma_{y}}{2} \quad ; W_{z}=\frac{\sigma_{z}}{2}
$$

Combining $\mathrm{SU}(2)_{\mathrm{W}}$ with $\mathrm{SU}(3)$ one is thus led to consider $\mathrm{SU}(6)_{\mathrm{W}}$, strong as the natural candidate for this "relativistic spin symmetry". In a constituent quark model, the generators of this algebra would then be written

$$
\begin{aligned}
& W^{i}=\sum_{q, \bar{q}} \frac{\lambda^{i}}{2}+\frac{\bar{\lambda}^{i}}{2} \\
& W_{x}^{i}=\sum_{q, \bar{q}} \frac{\lambda^{i}}{2} \beta \frac{\sigma_{x}}{2}+\frac{\bar{\lambda}^{i}}{2} \beta \frac{\sigma_{x}}{2} \\
& W_{y}^{i}=\sum_{q, q^{2}} \frac{\lambda^{i}}{2} \beta \frac{\sigma_{y}}{2}+\frac{\bar{\lambda}^{i}}{2} \beta \frac{\sigma_{y}}{2} \\
& W_{z}^{i}=\sum_{q, \bar{q}} \frac{\lambda^{i}}{2} \frac{\sigma_{z}}{2}+\frac{\bar{\lambda}^{i}}{2} \frac{\sigma_{z}}{2}
\end{aligned}
$$

$\sum_{q, \bar{q}} \alpha_{z}$, which, for free quarks, is the generator of Lorentz boosts in the $\vec{z}$ direction. This last property of $\mathrm{SU}(6)_{\mathrm{W}}$, strong for free quarks suggests that perhaps the physical $\mathrm{SU}(6)_{\mathrm{W}}$, strong might be an appropriate algebra for particles moving along the 2 axis, and, in particular, for decay amplitudes or for collinear processes. It should be pointed out, however, that this is an assumption which is not really well motivated. It allows us to classify moving states knowing their rest frame classification. In the rest frame both quark spin and:W spin are well defined and one finds that for

$$
\begin{aligned}
\text { quarks } & \vec{W}=\vec{S} \\
\text { antiquarks } & W_{z}=S_{z} \\
W_{x, y} & =-S_{x, y}
\end{aligned}
$$


where $\vec{S}$ starlds for ordinary quark spin. These relations foilow trivially from $\beta q=+q$ and $\beta \bar{q}=-\bar{q}$. As is well known, one finds from Eqso (1.4) the so-called W-S flip 21), e.g.,

$$
\begin{array}{ll} 
& \left|\rho\left(J_{z}=1\right)\right\rangle \sim\left|w=1, w_{z}=1\right\rangle \\
& \\
\text { but } \quad & \left|\rho\left(J_{z}=0\right)\right\rangle \sim-\left|w=0, w_{z}=0\right\rangle \\
\text { while } & |\pi\rangle \sim-\left|w=1, w_{z}=0\right\rangle
\end{array}
$$

Under Lorentz boosts in the $\hat{z}$ direction, $I$ does not remain a good quantum number but $I_{z}$, will continue to be conserved (as well as $W_{z}$, of course).

Even though $\mathrm{SU}(6)_{W}$ appears to be an approximate syrametry for the classification of one particle (moving) states it need not be a symnetry of the whole Hilbert space. An illustrative example of a similar situation is found in the case of the hydrogen atom. The energy levels of the $H$ atom possess an extra degeneracy beyond that of rotational invariance: states of different angular momentum but the same principal quantum number have the same energy. It may be shown that the generators of an $O(4)$ symmetry commute with the Hamiltonian of the $H$ atorn 22): however, the scattering of bound. states does not possess this symetry. Thus $O(4)$ is only useful for analyzing the spectrum of isolated states and not for the dynamics of particle interactions.

If, however, one does assume $\operatorname{sid}(6)_{\mathrm{W}}$, strong for collinear processes such as three-point functions ["narve" su( 6$)_{\mathrm{W}}$, one has separate conservation of $L_{z}$ and $W_{z}$. It is precisely this restriction which appears to be violated in nature 15$), 16), 18)$.

Hence, we can only conclude that the generators of $\mathrm{SU}(6)_{\mathrm{W}, \text { strong }}$ approximately comnute with the strong interaction Hamiltonian when sandwiched between one-particle states, and that $\mathrm{SU}(6)_{\mathrm{W}}$, strong is not, even approximately, a vertex symnetry. For this reason phenomenological models were proposed several years ago with the intention of relaxing $\mathrm{SU}(6)_{\mathrm{W}, \text { strong }}$ symmetry somewhat. 
It was shown by Carlitz and Kislinger ${ }^{23)}$ that one can construct $\mathrm{SU}(6)_{\mathrm{W}}$ invariant vertices by working with states of quark spin but allowing an additional quark-antiquark pair to be produced with $\mathrm{L}_{z}=0$, $\mathrm{P}=\mathrm{C}=+$, and internal quantum numbers of an $\mathrm{SU}(3)$ singlet. This pair shares its quarks among the two outgoing particles in the decay. On the basis of this picture, it was guessed that a useful modification of naIve $\mathrm{SJ}(6)_{\mathrm{W}}$ would result from allowing the pair to have $I_{z}= \pm 1$ as well as $\mathrm{L}_{z}=0$ 15). This model has come to be called the " ${ }^{2}{ }_{0}$ picture", 14),15) since the $q \bar{q}$ pair is assumed to have all the quantum numbers of the vacuum. Actually the name is somewhat nisleading, since ${ }^{3} \mathrm{P}_{0}$ implies a decinite relation between $I_{z}= \pm 1$ and $I_{z}=0$. In contrast, the model assumes the relative admixtures of these two states to be arbitrary。

In various practical applications ${ }^{14)-19)}$ it has been shown that the ${ }^{3} \mathrm{P}_{0}$ picture is equivalent to breaking only those naive $\mathrm{Su}(6)_{W}$ relations which link different final relative orbital momenta $\&$ between the two resonance decay products. Those naIve $S U(6)_{W}$ predictions referring to a given $l$ are preserved. This scheme is the " $l$ broken SU(6) W" referred to in the Introduction. As we shall show, the simplest place where $\ell$. broken $\mathrm{SU}(6)_{\mathrm{W}}$ is only a special case of (and not equivalent to) the ${ }^{3} \mathrm{P}_{\mathrm{O}}$ picture is in $\mathrm{I}=2 \rightarrow \mathrm{L}=1+$ pion decays.

Two subgroups of SU(6)W, strong have also been applied to hadron decays. These are the coplanar 15), 24) and chiral 12), 13) versions of $\mathrm{U}(3) \times \mathrm{U}(3)$.

In the picturesque language of constituent quarks, the possibility of transverse motion of these quarks inside a hadron suggests that one studies the subset of the generators (1.3) commuting both with a Lorentz boost along the $z$ axis and a transverse boost (say $\alpha_{X}$ in the free quark model). This gives rise to the coplanar $\mid \underline{U}(3) \times U(3)] \beta \sigma_{y}$ first discussed by Dashen and Gell-Mann 25). This symmetry was explicitly applied to decays in Ref。 24) and was found to be weaker than the ${ }^{3} \mathrm{P}_{\mathrm{O}}$ picture but consistent with it.

The chiral version of $U(3) \times U(3)$ strong generated by $w^{i}$ and $\mathrm{w}_{z}^{i}$ is somewhat better rnotivated theoretically since in any interacting quark model these generators should always commute with boosts along the 2 axis. On the other hand, from the current quark point of view, as will be seen in the next Section, the chiral $U(3) \times U(3)$ currents is well established 
while the existence of tensor currents, which can be constructed in a current quark model and used to exterid the chiral algebra to an $S U(6)$ w, currents algebra, requires an additional assumption. Hence it is of some interest to study the implications and the breaking pattern of the chiral $\mathrm{SU}(3) \times \mathrm{SU}(3)$ strong subalgebra of $\mathrm{SU}(6)_{\mathrm{W}, \text { strong }} \cdot$

\section{CURRENT QUARSS}

To discuss current-quark algebraic structures let us start from the well-kmow chiral $\mathrm{SU}(3) \times \mathrm{SU}(3)$ algebra ${ }^{2)}$ of vector and axial vector charges

$$
\begin{aligned}
& F^{i}(t)=\int d^{3} x \tilde{F}_{0}^{i}(x) \\
& F^{i 5}(t)=\int d^{3} \times \tilde{F}_{0}^{i 5}(x)
\end{aligned}
$$

where the current densities $\mathcal{F}_{\mu}^{i}(x)$ and $\mathcal{F}_{\mu}^{i 5}(x)$ are well defined measurable physical operators in weak and electromagnetic transitions. In a "current-quark" model; one would write these vector and axial vector current densities as

$$
\begin{aligned}
& F_{\mu}^{i}(x)=\bar{q}(x) \gamma_{\mu} \frac{\lambda^{i}}{2} q(x) \\
& F_{\mu}^{i s}(x)=\bar{q}(x) \gamma_{\mu} \gamma_{s} \frac{\lambda^{i}}{2} q(x)
\end{aligned}
$$

From these expressions and the canorical anticonmutation relations of the quark fields one derives the equal time commutators:

$$
\begin{gathered}
{\left[F^{i}(t), F^{j}(t)\right]=i f_{i j k} F^{k}(t) ;\left[F^{i}(t), F^{j s}(t)\right]=i f_{i j k} F^{k s}(t)} \\
{\left[F^{i s}(t), F^{j s}(t)\right]=i f_{i j k} F^{k}(t)}
\end{gathered}
$$

This $\mathrm{SU}(3) \times \mathrm{SU}(3)$ algebraic structure is then assumed for physical vector and axial vector charges independently of any underlying quark structure. As is well known, this algebra leads, with the help of PCAC, to Adler-Weisberger sun rules. Insofar as they have been tested these are remarkably successful. In view of this, one can attempt, following Dashen and Gell-Mann, to enlarge the algebra. With the equal time 
commutation relations of the quark model one can clearly define an $U(12)$ algebra generated by 144 charges defined as integrals over the densities

$$
\bar{q}(x) \Gamma \frac{\lambda^{i}}{2} q(x)
$$

where $\Gamma$ is one of the 16 Dirac covariants. All these current densities are in principle directly or indirectly measurable: for example, although nothing in nature seems to be coupled directly to a tensor current $\mathcal{F}_{\mu \nu}^{i}(x)$, represented in the quark model by $\bar{q}(x) \sigma_{\mu \nu}\left(\lambda^{i} / 2\right) q(x)$, this current nevertheless appears on the right-hand side of commutators of more directly observable operators, e.g., in $\left[F_{\mu}^{i}(x), \partial^{\nu} F_{\nu}^{i 5}(y)\right]_{x_{0}}=y_{0} \cdot$ In this sense all 144 current densities could be meaningful physical operators provided, of course, that the algebraic structure of the current quark model can be abstracted that far.

It is important. to realize that contrary to the situation in the constituent quark approach one has here an algebraic structure which is generated by well defined operators: they are integrals over local current densities and their Lorentz transformation properties are given "by definition". Let us go in particular to the infinite momentum frame. As usual we define as "good" operators those charges whose matrix elements do not vanish when taken between finite mass states with infinite momentum along, say, the 2 direction 6$)$. There are 35 independent good operators *) whose quark model expressions are given by

$$
\begin{aligned}
& F^{i}(t)=\int d^{3} x F_{0}^{i}(x)=\int d^{3} x q^{+}(x) \frac{\lambda^{i}}{2} q(x) \\
& F_{x}^{i}(t)=\int d^{3} x F_{23}^{i}(x)=\int d^{3} x q^{+}(x) \beta \sigma_{x} \frac{\lambda^{i}}{2} q(x) \\
& F_{y}^{i}(t)=\int d^{3} x F_{31}^{i}(x)=\int d^{3} x q^{+}(x) \beta \sigma_{y} \frac{\lambda^{i}}{2} q(x) \\
& F_{z}^{i}(t)=\int d^{3} x F_{3}^{i 5}(x)=\int d^{3} x q^{+}(x) \sigma_{z} \frac{\lambda^{i}}{2} q(x)
\end{aligned}
$$

*) Properties of good currents and indeed the Melosh transformation itself may be reformulated on a light-like surface. This is perhaps a more exact expression of the infinite momentum limit. 
These charges generate at infinite momentum an SU(6) algebra which we will call $\mathrm{SU}(6)_{\mathrm{W}}$, currents ${ }^{*}$ The algebraic similarity between these $F_{\alpha}^{i}$ or "current-quark generators" and the $w_{\alpha}^{i}$ or "constituent quark generators" defined in the previous section is now obvious. It is, however, extremely important not to confuse or to identify too quickly the two sets of generators of these $S J(6)_{W}$ algebras. The $F_{\alpha}^{i}$ 's are explicitly integrals over local expressions with well defined Lorentz transformation properties, but they have not been assumed to commute with the strong interaction Hamiltonian. The $w_{\alpha}^{i} i s$, on the other hand, are supposed to commute approximately with the strong interaction Hamiltonian at least between one-particle states but there is no a priori reason why they should be integrals over local measurable densities. (In fact, as we shall see below, one finds that even in the free quark model 11) the $F_{\alpha}^{i}$ 's do not commute with the "strong interaction" Hamiltonian and that the $w_{\alpha}^{i}$ 's are not local charges.) Hence one has two sets of possibly distinct operators which generate the same $\mathrm{SU}(6)_{W}$ algebraic structure. A fundamental question is of course the relation, if any, between the two sets of generators. From the successes of the conserved vector current hypothesis (CVC) we are forced to identify the $w^{i}$ and the $F^{i}$, i.e,, the generators of ordinary $\mathrm{SU}(3)$ of strong interactions and the integrals of the time components of the vector currents. To identify the full sets of generators of $\mathrm{SU}(6)_{\mathrm{W}}$, strong and $\mathrm{SJ}(6)_{\mathrm{W}}$, currents would, however, be incorrect. A strong objection to such an identification comes from the Adler-Weisberger relation which, when saturated with resonances necessarily implies representation mixing, i.e., particles which belong to different $\mathrm{SU}(6)_{\mathrm{W}, \text { strong }}$ representations are connected by the axial charge and thus at least a piece of their wave function must lie in the same representation of $\mathrm{SJ}(6)_{\mathrm{W}}$, currents ${ }^{\circ}$ One could argue, perhaps, that if the Adler-Weisberger sum rule is saturated with resonances $(N, \Delta)$ belonging to the same $\operatorname{SiJ}(6)_{W}$, strong multiplet, the result obtained for $-G_{A} / G_{V}, 5 / 3$, is not too far removed from the correct value. Dashen and Gell-Mann 5 ) have shown, however, that in the same approximation, i.e., classifying the nucleon

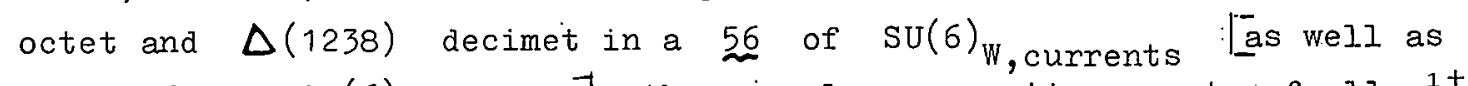
in a 50 of $\left.\mathrm{SJ}(6)_{\mathrm{W}, \text { strong }}\right]$ the anomalous magnetic moments of all $\frac{1}{2}^{+}$ baryons and the magnetic transitions between the $\frac{1}{2}^{+}$octet and $\frac{3}{2}^{+}$decimet are predicted to vanish. This is clearly unacceptable. One arrives at the conclusion that there must be sizeable representation mixing and thus that the generators of the two $\mathrm{SU}(6)_{\mathrm{W}}$ algebras cannot be identified. This being the case one may try to relate the two algebras by a unitary transformation. In fact there is no compelling argument in favour of such an 
assumption: it is motivated by the feeling that the similarity in these algebraic structures is a fundamental property of the hadronic world. If to the quantum numbers corresponding to particle classifications under either $\mathrm{SU}(6)_{\mathrm{W}}$ one adds all necessary quantum numbers to obtain two bases of the Hilbert space, a unitary transfomation relating one of these bases to the other must exist. This does not imply. that the generators of the two SU $(6)_{W}$ algebras are, by themselves, related through a unitary transformation but it makes such an assumption somewhat more plausible. Furthermore Melosh 11) showed recently that in the free quark model such a unitary transformation does in fact exist. We will postulate that such a transformation exists in general and write

$$
W_{\alpha}^{i}=V F_{\alpha}^{i} V^{-1}
$$

where.$V$ is unitary and will be called the Melosh transformation. This transformation. $V$ thus expresses the general idea that hadrons can be simultaneously. described as simple. constituent quark states where strong interactions are concerned and as rather complicated structures when currents are involved. It systematizes in a compact way all $\mathrm{SU}(6)_{\mathrm{W} \text {, currents }}$ representation mixing for all hadrons. To make this last point clear let us recall that $W_{z}^{0}$ and $F_{z}^{\circ}$ act like quark spin operators for constituent and current quarks respectively. Both operators commute with $J_{z}$ and thus make it possible to define "orbital" angular momenta 26) by

$$
L_{z}(W)=J_{z}-W_{z}^{0}
$$

and

$$
L_{z}(F)=J_{z}-F_{z}^{0}
$$

We may then classify states according to their $\mathrm{SU}(6)_{\mathrm{W}}$ quantum numbers and angular momentum. For example the $\mathrm{SU}(6)_{\mathrm{W}}$, strong classification of the lowest lying mesons would be $35 \oplus \stackrel{1}{\sim}, I_{z}(W)=0$ while the first excited states would be $35 \oplus: 1, I_{z}(W)=-1,0,+1$.

The Melosh transfornation $V$ must obviously be invariant under rotations around. the $\hat{z}$ axis $i . e .,\left[\bar{v}, J_{z}\right]=0$. Let us suppose that $\left[\mathrm{V}, \mathrm{F}_{z}^{\mathrm{O}}\right] \neq 0$ so that $\mathrm{F}_{z}^{0}$ and $\mathrm{W}_{\mathrm{z}}^{0}$ are different operators. A state with a simple constituent quark spin structure will then in general be expected to correspond to a mixture of different current quark spin states. Similarly, while the constituent orbital angular momentum $I_{z}(W)$ will be simple, one will have a mixture of current orbital angular momenta $I_{z}(F)$ since $J_{z}=W_{z}^{0}+I_{z}(W)=F_{z}^{0}+I_{z}(F)$, and $J_{z}$ conmutes with the Melosh transformation. 
To conclude this section on current quarks we recall the explicit construction of the Melosh transfornation in the free quark model. Such a model is defined by the Hamiltonian

$$
H_{\text {free }}=\int d^{3} x q^{+}(x)\{-i \vec{\alpha} \cdot \vec{\partial}+\beta m\} q(x)
$$

The generators of $\mathrm{SU}(6)_{\mathrm{W}}$, currents are given, in this model, by the expressions appearing in Eqs. $(2.6)$. They do not commute with $\mathrm{H}_{\text {free }}$ and hence cannot be identified with the generators of $\mathrm{SU}(6)_{\mathrm{W}}$, strong ${ }^{\cdot}$ In this sense; the free quark model, although trivial, exhibits some of the complexity of the full problem. As shown by Melosh with

$$
V_{\text {free }}=\exp \left(i Y_{\text {free }}\right)
$$

and $\quad Y_{\text {free }}=\frac{1}{2} \int d^{3} x q^{+}(x) \arctan \left(\frac{\vec{\partial}_{1} \cdot \vec{\partial}_{1}}{m}\right) q(x)$

one finds, through Eq. (2.7), the following set of operators

$$
\begin{aligned}
& W_{\text {, free }}^{i}=F_{\text {, free }}^{i} \\
& W_{x, \text { free }}^{i}=F_{x, \text { free }}^{i}+\int d^{3} x q^{+}(x) \frac{1}{x}\left\{\frac{1}{1+x} \frac{\vec{\gamma}_{1} \vec{\partial}_{1}}{m}-i\right\} \gamma_{s} \frac{\partial_{x}}{m} \frac{\lambda^{i}}{2} q(x) \\
& W_{y, \text { free }}^{i}=F_{y, \text { tree }}^{i}+\int d^{3} x q^{+}(x) \frac{1}{k}\left\{\frac{1}{1+x} \frac{\vec{\gamma}_{1} \cdot \vec{\partial}_{1}}{m}-i\right\} \gamma_{s} \frac{\partial_{y}}{m} \frac{\lambda^{i}}{2} q(x) \\
& W_{z, \text { free }}^{i}=F_{z, \text { free }}^{i}+\int d^{3} x q^{+}(x) \frac{1}{x}\left\{\frac{1}{1+x} \frac{\vec{\gamma}_{1} \cdot \vec{\partial}_{1}}{m}-i\right\} \sigma_{z} \frac{\vec{\gamma}_{1} \vec{\partial}_{1}}{m} \frac{\lambda^{i}}{2} q(x)
\end{aligned}
$$

where

$$
K=\left[1+\left(\frac{\vec{\delta}_{1} \cdot \vec{\partial}_{1}}{m}\right)^{2}\right]^{1 / 2}
$$

These transformed operators can be shown to conmute with $H_{\text {free }}$ and are thus appropriate as $\mathrm{SU}(6)_{\mathrm{W}}$, strong generators. "Some of the properties of the Melosh transformation are worth pointing out. The first remarkable property of $v_{\text {free }}$ is that it is non-local in the transverse directions and hence, as shown explicitly by Eqs. (2.11), the symmetries of the Hamiltonian are generated by non-local operators! Introducing the (current) quark spin and orbital angular momentum operators 


$$
\begin{aligned}
& \Sigma_{i, \text { free }}=\int d^{3} x q^{+}(x) \frac{\sigma_{i}}{2} q(x) \\
& L_{i, \text { free }}=i \epsilon_{i j k} \int d^{3} x x_{k} q^{+}(x) \partial_{j} q(x)
\end{aligned}
$$

we can describe the properties of $Y_{\text {free }}$ under $\mathrm{SU}(6)_{\mathrm{W}}$, currents $\times 0(2)$ as follows: $Y_{\text {free }}$ is the $\mathrm{SU}(3)$ singlet member of a 35 with $\Delta J_{z}=0$,

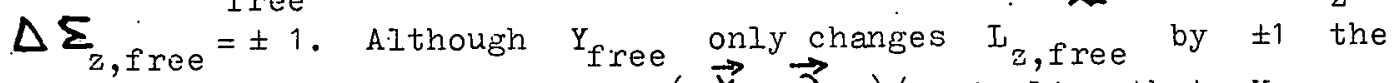
presence of the power series in $\left(\vec{\gamma}_{\perp} \cdot \vec{\partial}_{\perp}\right) / \mathrm{m}$ implies that $Y_{\text {free }}$ can mix essentially all total angular momenta. Let us note also that the $w_{\alpha}^{i}$ have simple algebraic properties under $\mathrm{SU}(6) \mathrm{W}$, currents $\times O(2)$ : they transform as the sum of a 35 with $\Delta S_{z}=\Delta I_{z}=0$ and of a 35 with $\Delta S_{z}=-\Delta I_{z}= \pm 1$.

\section{THE MODEL AND ITS APPLICATIONS TO MESON RESONANCES}

a) The $\mathrm{SU}(6) \mathrm{W}_{\mathrm{W}}\left(\Delta \mathrm{I}_{z}=0, \pm 1\right)$ model

Given the existence of a unitary transformation between constituent quarks and current quarks we may now proceed, with the heip of PCAC, to a "theory" of pionic transitions. As hadrons do seem to fall into irreducible representations of $\mathrm{SU}(6)_{\mathrm{W}}$, strong, this algebra is an approximate symmetry of the strong interaction harniltonian (at least for one-particle states).

This does not imply : that $\left.\mathrm{SU}_{(6)}\right)_{\mathrm{W}}$, strong can be considered as an approximate symnetry in the whole Hilbert space. Indeed, as discussed in Section 1 one finds that $\mathrm{SU}(6)_{\mathrm{W}}$, strong is totally unacceptable as a vertex symmetry. on the other hand, the PCAC relation

$$
\partial^{\mu} F_{\mu}^{i s}=c \phi^{i} \quad(i=1, \ldots 8)
$$

allows us to write pionic three-point functions as follows:

$$
\left\langle B \pi^{i} \mid A\right\rangle \propto\left(\frac{m_{B}^{2}-m_{A}^{2}}{c}\right)\left\langle B\left|F^{i 5}\right| A\right\rangle
$$

Thus, PCAC reduces those hadronic three-point functions involving at least one pseudoscalar meson to the matrix elements oi the axial charge between one-particle states for which $\mathrm{SU}(6)_{\mathrm{W}}$, strong is expected to be a good 
approximate symmetry. But the axial charges $I^{\text {i5 }}$ are of course generators o. $\mathrm{SU}(6)_{\mathrm{W}}$, currents and they are assumed to be related by the (unitary) . Melosh transformation to the $\mathrm{SJ}(6)_{\mathrm{W}}$, strong generators. Hence any assumption about the Melosh transformation $V$ will reflect itself in pionic decays of hadrons. Let us formalize this connection by defining the Melosh transformation as the unitary transformation of the Hilbert space which relates the constituent quark representation [i.e., in $\mathrm{SU}(3) \times \mathrm{SU}(3)$ strong

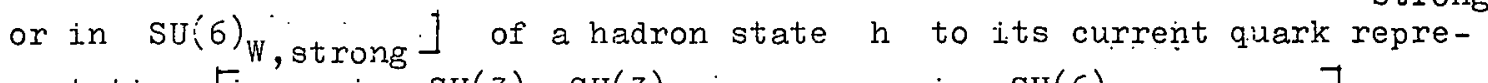
sentation [i.e., in $\mathrm{SU}(3) \times \mathrm{SU}(3)_{\text {currents }}$ or in $\left.\mathrm{SU}(6)_{\mathrm{W}, \text { currents }}\right]$ :

$$
\mid h \text {; current quark }\rangle=V \mid h \text {; constituent quark }\rangle
$$

The matrix elements of the axial charges $\mathrm{F}^{\mathrm{i} 5}$, given by.

$$
\text { 〈h'; currentquark } \left.\left|F^{i s}\right| h \text {; currentquark }\right\rangle
$$

can then be rewritten as

$$
\left\langle h^{\prime} \text {; constituentquark }\left|V^{-1} F^{i 5} V\right| h \text {; constituentquark }\right\rangle
$$

As discussed in the previous section, in the iree quark model, where free was explicitly constructed by Melosh, one finds that the transformed axial charge has a remarkably simple algebraic structure: it transforms,

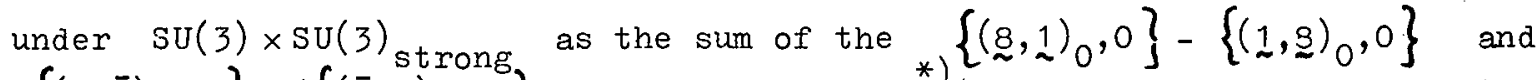
$\left\{(3, \overline{3})_{1},-1\right\}-\left\{(\overline{3}, 3)_{-1}, 1\right\}$ representations $\left.{ }^{*}\right)$ : Equivalently, the transformed axial charge transforms, under SU(6) W, strong, ' as the sum of the $\left\{35, I_{z}=0\right\}$ and $\left\{35, I_{z}= \pm 1\right\} \quad$ representations.

We will assume in this paper that these simple algebraic properties of the transformed free quark axial charge can be extended to the physical axial charge. This is of course an extreinely strong assumption: it can be somewhat notivated by the following argument.

As long as the Melosh transformation is bilinear in quark fields these algebraic properties of the axial charge are in fact the most general ones. Indeed, for the axial charges belonging to $\mathrm{SU}(3)$ octets with $\Delta \mathrm{J}_{\mathrm{z}}=0$, a Melosh transformation bilinear in quarks which is an $\mathrm{SU}(3)$ singlet and

*) We have used the standard notation $\left\{(\underset{\sim}{A}, B)_{S_{z}}, I_{z}\right\}$ to label irreducible representations of $\mathrm{SU}(3) \times \mathrm{SU}(3)$ at infinite momentum along the 2 axis, corresponding to helicity $\mathrm{S}_{z}+\mathrm{I}_{z}$ and to total quark spin $\mathrm{S}_{z}$. 
commutes with $J_{z}$, can only transform them to octets with $\Delta S_{z}=0$, and $\Delta \mathrm{S}_{\mathrm{z}}= \pm 1$. This is equivalent to the $\mathrm{SU}(3) \times \mathrm{SU}(3)$ strong or $\mathrm{SU}(6)_{\mathrm{W}}$, strong transformation properties of the free transformed axial charge. "The question then is of course why $V$ should be bilinear in quarks. We do not have any compelling argument in favour of this assumption except that it is the simplest way to connect two remarkable properties of hadronic physics: both currents and hadronic states seem. to behave in some sense like free quark currents and free quark states respectively. A bilinear Melosh transformation allows us to connect these two "free quark" approximations.

Before using Eq. (3.4) to compute pionic decays of hadrons we still have to connect real hadronic states to free constituent quark states. Although we know of no satisfactory prescription to make this connection, especially since we do not know the strong interaction Hamiltonian, it seems reasonable to assume that physical one-particle states can be constructed by acting with some operator $U$ on free quark states ${ }^{11)}$. The success of $\mathrm{SU}(6)_{\mathrm{W}}$, strong or its subgroup $\mathrm{SU}(3) \times \mathrm{SU}(3)$ strong in classifying hadronic states can then be ưnderstood if $U$ : is assumed to transform as an isU(6) $\mathrm{W}$, strong or $\mathrm{SU}(3) \times \mathrm{SU}(3)$ strong singlet.

To summarize, our model for pionic decays is based on the following

assumptions

1) PCAC;

2). "free constitient quark" classification of hadrons in $S U(6)_{W}$, strong or : $\mathrm{SU}(3) \times \mathrm{SU}(3)$ strong (U transforms as a singlet)

3) $\mathrm{V}^{-1} \mathrm{~F}^{\mathrm{i}} \mathrm{V}$ transforms as the sum of the $\left\{(8,1)_{0}, 0\right\}-\left\{(1,8)_{0}, 0\right\}$ and $\left\{(\bar{z}, \overline{3})_{1},-1\right\}-\left\{(\bar{z}, 3)_{-1}, 1\right\}$ representations of $\mathrm{SU}(3) \times \mathrm{SU}(3)$ strong or equivalently as the sum of the $\left\{35, \mathrm{~L}_{z}=0\right\}$ and $\left\{35, \mathrm{~L}_{z}= \pm 1\right\}$ representations of $\mathrm{SU}(\dot{\sigma})_{\mathrm{W}}$, strong ${ }^{\text {. }}$

In the remainder of this section we will refer to our model as the $\mathrm{SU}(6)_{\mathrm{W}}\left[\Delta \mathrm{I}_{\mathrm{z}}=0, \pm 1\right]$ módel.

b) Classification according to $\mathrm{U}(3) \times \mathrm{U}(3)$ and $\mathrm{SU}(6) \mathrm{W}_{\mathrm{W}}$

We shall need the reduction of the $(6, \sqrt[6]{6})$, I states of $U(6) \times U(6) \times 0(3)$ to combinations of $\mathrm{U}(3) \times \mathrm{U}(3) \times O(2)_{\mathrm{L}_{\mathrm{Z}}}$ or $\mathrm{SU}(6)_{\mathrm{W}} \times O(2)_{\mathrm{I}_{\mathrm{Z}}}$ states. Once we reduce the states of given quark spin $S$ and $z$ component $S_{Z}$, the extension to states of arbitrary $I$ will be trivial. 
The relation between quark-spin and $W$-spin states was already pointed out in section 1. In the discussion of $[U(3) \times U(3)]_{S_{2}}$ we need only recall that $\sigma_{z} q=S_{z} q$ while $\sigma_{z} \bar{q}=-S_{z} \bar{q}$. Then Table Ia) shows the necessary relations between the rest symmetry, chiral $U(3) \times U(3)$, and $S U(6)_{W}$ classifications. For simplicity we consider only octets, avoiding the discussion of nonet couplings except in the context of phenomenological interpretations.

States o: arbitrary. I with given helicity are then expressed in terms of those in Table Ia) via clebsch-Gordan coefficients.

$$
|J S L \lambda\rangle=\sum_{S_{z}+L_{z}=\lambda}\left(S S_{z} L L_{z} \mid J \lambda\right)\left|S S_{z}\right\rangle
$$

The $J^{P C}$ values of the specific states considered here are shown in Table $1 \mathrm{~b}{ }^{*}$ ).

c) Predictions for specific processes

The reduced matrix elements within a given set of decays $I \rightarrow L^{1}+\pi$ in chiral $U(3) \times U(3)$ may be labelled by the initial $I_{z}^{i}$, the final $I_{z}^{f}$, and the total helicity $\lambda$. In addition, for reduced matrix elements of three octets $(8,1) \rightarrow(8,1) \otimes(8,1)$ and $(1,8) \rightarrow(1,8) \otimes(1,8)$, the relative phase of the two depends on the product of the charge parities of $A(I)$, $B\left(L^{\prime}\right)$ and $T$. In practice for the cases we consider, this leads to an extra. degree of freedom in the $\mathrm{L}_{z}^{i}=\mathrm{I}_{z}^{f}=\lambda=1$ amplitudes, which, will be expressed via a parameter $\mu$.

Since chiral $\mathrm{SU}(3) \times \mathrm{SU}(3)$ is the weakest of the symmetries we consider, our Tables will give its predictions, with a simple "dictionary" at the end of each table for converting to the stronger symmetries.

$I_{z}^{f} J_{Z}^{i}$ The reduced matrix elements in chiral $\mathrm{SU}(3) \times \mathrm{SU}(3)$, called $a^{L_{z} L_{z}}$, are normalized so that in the $\operatorname{SU}(6)_{W}\left(\Delta I_{z}=0, \pm 1\right)$ limit

*) It is necessary to take some care in choosing a consistent set of conventions for the behaviour of $\left\{[\mathrm{U}(3) \times \mathrm{U}(3)]_{\mathrm{S}_{\mathrm{z}}}, \mathrm{I}_{\mathrm{z}}\right\}$ representations under charge conjugation and parity. For example, one $\left\{(8,1)_{0}-(1,8)_{0}, 0\right\}$ representation represents a pion with $\mathrm{C}=+1$ and another, different $\left\{(8,1)_{0}-(1,8)_{0}, 0\right\}$ representation, an helicity zero $B$ with $\mathrm{C}=-1$. 


$$
a_{\lambda}^{L_{2}^{f} L_{z}^{i}}=a^{L_{z}^{f} L_{z}^{i}}
$$

except for reduced chiral $S U(3) \times S U(3)$ matrix elements which vanish in this limit $(\mu=0)$. The helicity amplitudes $g \lambda$ for $A(I) \rightarrow B\left(I^{\prime}\right)+\pi$ in $\mathrm{SU}(6)_{\mathrm{W}} \quad\left[\Delta \mathrm{I}_{\mathrm{z}}=0, \pm 1\right]$ are

$$
\begin{aligned}
& g_{\lambda}=\sum_{L_{z}^{\prime}, L_{z}^{i}} \xi^{A}\left(\lambda-L_{z}^{i}\right)\left(S^{A} \lambda-L_{z}^{i} L L_{z}^{i} \mid J^{A} \lambda\right) \\
& \times \xi^{B}\left(\lambda-L_{z}^{f}\right)\left(S^{B} \lambda-L_{z}^{f} L^{\prime} L_{z}^{f} \mid J^{B} \lambda\right) \\
& \times\left\{\begin{array}{cc}
35 & 35 \\
\left(8,2 w^{B}+1\right) & (8,3)
\end{array} \mid\left(\underset{\left.8,2 w^{A}+1\right)}{35}\right\}_{\alpha_{1}}\left\{\begin{array}{cc|c}
8 & 8 & 8 \\
B & \pi & A
\end{array}\right\}_{\alpha_{2}}\right. \\
& \times\left(W^{B} \lambda-L_{z}^{f} 1 L_{z}^{f}-L_{z}^{i} \mid W^{A} \lambda-L_{z}^{i}\right) a^{L_{z}^{f} L_{z}^{i}}
\end{aligned}
$$

The first two Clebsch-Gordan coefficients in Eq. (3.7) are those coming from $\mathrm{Eq}$. (3.5). The next term is an $\mathrm{SU}(3)$ scalar factor $[\mathrm{a}$ clebschGordan coefficient of $\mathrm{SU}(6)$ 27) antisymmetric coupling of $35 \rightarrow 35 \otimes 35$; it is chosen so that the $\mathrm{SU}(2)$ scalar factor [Clebsch-Gordan coefficient of SU(3) 28)] denotes the proper: a type or $f$ type coupling as dictated by the product of charge parities of $A, \cdots$ and $\pi$ :

$$
\begin{aligned}
& G(A) G(B) G(\pi)=+: \alpha_{2}=" d \text {-type" } \\
& b(A) G(B) b(\pi)=-: \alpha_{2}=" f \text {-type" }
\end{aligned}
$$

In the isoscalar factors, we shall take the specific isomultiplets $A=B=K$ (the $I=\frac{1}{2}, \quad Y=+1$ states) for convenience. The phases $\xi^{A}$ and $\xi^{B}$ arise from the relation between quark spin and $W$ spin states [see, e.g., $\mathrm{Eq} .(1.5)]$, and are

$$
\begin{aligned}
\xi^{A, B}\left(S_{z}\right) & =+1: & S_{z}=+1 \\
& =-1: & \text { otherwise }
\end{aligned}
$$

Finally, the last Clebsch-Gordan coefficient in Eq. (3.7) expresses W spin conservation. Note that the value of $w_{z}$ associated with the pion $(w=1)$ 
need not be zero. It is zero only in the case of "naive" SU(6) $W$, where $\Delta \dot{I}_{z}=I_{z}^{f}-I_{z}^{i}=0$.

$$
\begin{aligned}
& \text { The Tables are: } \\
& \text { Table II : } I=1 \rightarrow I=0 \\
& \text { Table III: } I=2 \rightarrow I=0 \\
& \text { Table IV : } \mathrm{I}=1 \rightarrow \mathrm{I}=1 \\
& \text { Table V. } \mathrm{I}=2 \rightarrow \mathrm{L}=1 .
\end{aligned}
$$

We shall discuss the relations among various symmetries implied by these results in the next section. Meanwhile it is helpful to point out where comparisons with data may be made.

\section{d) Applications to experiment}

The case of. $I=1 \rightarrow I=0$ decays has. already been studied in a formalism equivalent to $\operatorname{SU}(6)_{\mathrm{W}}\left(\Delta \mathrm{I}_{\mathrm{z}}=0, \pm 1\right){ }^{15)}$. The $\Delta \mathrm{I}_{\mathrm{z}}= \pm 1$ transitions were found to be dominant, as measured in the $B \rightarrow \omega \pi$ decay. Recently these fits are being repeated with kinematic factors appropriate to the use of $\mathrm{PCAC}{ }^{29)}$; qualitative features are expected to remain the. same.

Present information about $I=2 \rightarrow I=0$ decays allows one to check some predictions of Table II. . It is useful to rewrite these predictions in terms of the $l=1$ and $l=3$. amplitudes $a_{P}$ and $a_{F}$. (see the "dictionary" at the botton of the mable). One then obtains the results shown in Table VI. We quote here only partial width predictions. No decays $\left(2^{- \pm} \rightarrow 1^{--}\right)$have been observed as yet which would allow for comparison with helicity amplitude ratio predictions (in contrast to the case $B \rightarrow \boldsymbol{\omega} \pi$.) In constructing. Table VI we have used the fact that Table III refers to. "K" $\rightarrow$ "K" $\pi$. These values are then converted to specific processes using $\mathrm{SJ}(3)$ and (where necessary) a nonet ansatz for couplings. A convenient table for performing such conversions in general is given in Ref. 24).

The experimental predictions have been obtained by using the decay width $\Gamma(\mathrm{g}(1700) \rightarrow 2 \pi)=40 \mathrm{MeV}$, an approximate value suggested by a recent analysis 30 ) of the CERN-Munich data. The masses of decaying states are all taken at the idealized values of $1700 \mathrm{MeV}$ for the purpose of barrier factor calculations. We use zero-radius forms for these factors, as suggested by 
the successful application of $\mathrm{SJ}(3)$ to the known $I=2 \rightarrow I=0$ decays 31$)$. In fact, the interested reader is referred to that reference for a quotation of many SU(3) related results which will not be mentioned here, and is urged to recalculate any partial widths on the basis of exact masses and various barrier factors. The results quoted are somewhat sensitive to these choices. For example, the kinematic factor arising from the use of PCAO is such that

$$
\Gamma_{l}(A \rightarrow B r)=\frac{P\left(M_{A}^{2}-M_{B}^{2}\right)^{2}}{m_{A}^{2}} a_{l}^{2} C_{l}^{2}
$$

where $p$ is the final c.m. three-momentum, a are the reduced matrix elements $\left(a_{P}\right.$ and $a_{T}$ ror Table VI, as defined in Table III), and the squares of the ${ }^{c} l$ are shown in Table VI. Use of $\mathrm{Eq}$. (3.10) increases the prediction for $\Gamma(g \rightarrow \omega \pi)$ to $26 \mathrm{MeV}$ and that for $\Gamma\left(\omega_{3} \rightarrow \rho \pi\right)$ to $30 \mathrm{IIVV}$. These values are closer to the fit of Graham and Yoon, Ref. 31), but in our opinion the data do not yet allow one to decide conclusively in favour of such a kinematic factor in all cases. Note, for example, that the simple zero-radius $f$ wave barrier factor agrees with $\mathrm{SU}(3)$ fon the ratio $\mathrm{g} \rightarrow \mathrm{K} \overline{\mathrm{K}} / \mathrm{g} \rightarrow \pi \pi$, as it seems to do in a number of other cases.

The prediction for $\mathrm{A}_{3} \rightarrow \rho \pi(l=3)$ is notable. No evidence for this decay has been found so far either in $l=1$ or $l=3$.

For $L=1 \rightarrow I=1$ decays (Table IV) one expects one $f$ wave amplitude and two independent $p$ wave amplitudes in $\ell$ broken $S U(6)_{W^{*}}$ A partial wave decomposition of the helicity amplitudes for the various processes, shows however, that there are no purely $f$ wave decays. Hence simple iests analogous to those implied by Table III are not possible. Various processes which are purely $p$ wave depend on different combinations of $p$ wave amplitudes, However, one does have in $_{\mathrm{SU}}(6)_{W}\left[_{-} \Delta \dot{I}_{z}=0, \pm 1\right]$ or $l$ broken $\left.\mathrm{SU}(6)_{\mathrm{W}}\right]$ the following relations

$$
\frac{\tilde{\Gamma}\left[B \rightarrow \pi_{N}(980) \pi\right]}{\tilde{\Gamma}\left[D_{(y-\text {-likemixing })}^{\rightarrow} A_{1} \pi\right]}=\frac{4}{3}
$$


$-19-$

and

$$
\begin{aligned}
& \widetilde{\Gamma}\left[B \rightarrow A_{1} \pi\right]
\end{aligned}
$$

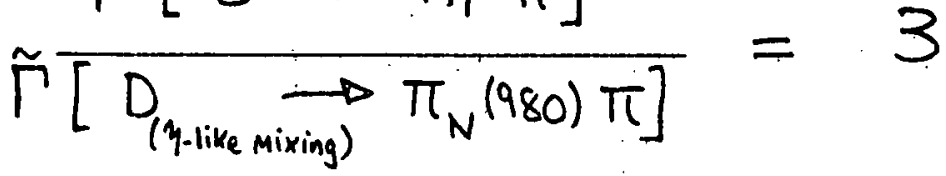

In both equations $\tilde{\Gamma}$ stands for the partial width with barrier factors removed. Since the $B$ is so close to $A_{1} \pi$ threshold, this leads to a rather small physical width for $B \rightarrow A_{1} \pi$ (a few $\mathrm{MeV}$, based on taking $20 \mathrm{MeV}$ for $\left.D \rightarrow \pi_{N} \pi\right)$. However, the strong intrinsic $B-A_{1}-\pi$ coupling should be observable in $B$ exchange processes, such as $\pi^{-} p \rightarrow A_{1}^{0} n$. To date we know of no firm evidence for this process, however.

Table $V$ contains a number of predictions for the $S$ wave partial widths in decays that involve higher partial waves as well. The most useful of these can be written as

$$
\begin{aligned}
& \tilde{\Gamma}_{l=0}\left[\rho\left(2^{-\infty}\right) \rightarrow A_{2} \pi\right]=a_{s}^{2} / 200 \\
& \tilde{\Gamma}_{l=0}\left[A_{3}\left(2^{-+}\right) \rightarrow f_{0} \pi\right]=3 a_{S}^{2} / 800 \\
& \tilde{\Gamma}_{l=0}\left[\rho^{\prime}\left(1^{-}, L=2\right) \rightarrow A_{1} \pi\right]=a_{S}^{2} / 240 \\
& \tilde{\Gamma}_{l=0}\left[w^{\prime}\left(1^{-}, L=2\right) \rightarrow B \pi\right]=a_{S}^{2} / 160
\end{aligned}
$$

If the $\mathrm{A}_{3}$ is a resonance, $\mathrm{f}_{0} \mathrm{~T}$ is its dominant decay mode. Then

$$
\frac{\Gamma_{l=0}\left[p\left(2^{-*}\right) \rightarrow A_{2} \pi\right]}{\Gamma_{l=0}\left[A_{3}\left(2^{-1}\right) \rightarrow f_{0} \pi\right]}=\frac{4}{3}
$$

implies a considerable value for the numerator. We hesitate to quote a predicted partial width since it is not clear whether the whole $l=0 \quad f_{0} \pi$ bump usually called $A_{3}$ is indeed resonant. It is interesting that there does seem to be evidence for a $\pi_{2}$ enhancement around $1700 \mathrm{MeV} 28$ ). A 
$-20-$

partial wave analysis of this effect, for example in the channel

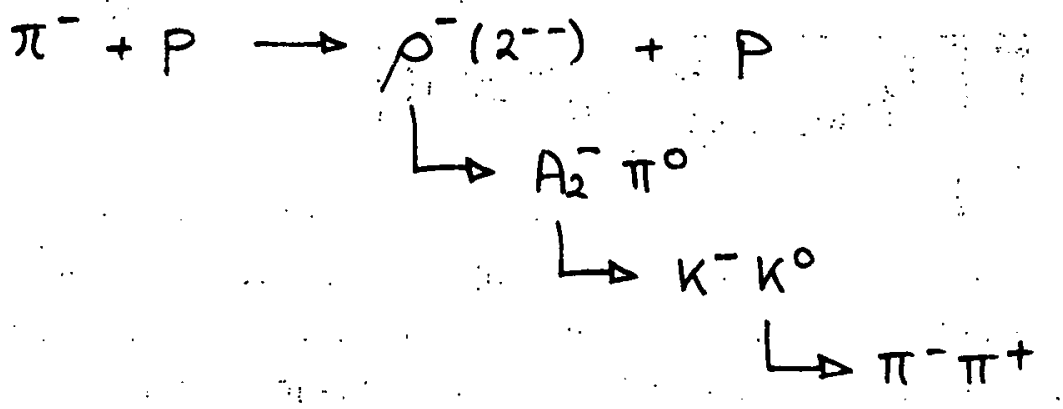

could oe performed using multi-particle spectrometers such as Omega at CRit.

There are two $d$ wave amplitudes in $\ell$ broken $\mathrm{SU}(6)_{\mathrm{W}}$ and three in $\mathrm{SU}(6)_{\mathrm{W}}\left[\Delta \mathrm{I}_{\mathrm{z}}=0, \pm 1\right]$. In $\ell$ broken $\mathrm{SU}(6)_{\mathrm{W}}$ but not in $\mathrm{SU}(6)_{\mathrm{W}}$ $\left.\left\lfloor\Delta I_{z}=0, \pm 1\right] !\right]$ the following relations hold:

$\tilde{\Gamma}\left[\omega\left(2^{-}\right) \rightarrow B \pi\right] \quad:=\frac{3}{160}\left(a_{0}^{\prime}\right)^{2}$

$\tilde{\Gamma}\left[A_{3}\left(2^{+}\right) \rightarrow D_{(8)} \pi\right]=\frac{1}{160}\left(a_{D}^{1}\right)^{2}$

$$
\tilde{\Gamma}\left[\rho\left(2^{-}\right) \rightarrow \pi_{N}(980) \pi\right]=\frac{1}{40}\left(a_{D}^{\prime}\right)^{2}
$$

$$
\tilde{\Gamma}\left[\rho^{\prime}\left(1^{-}, L=2\right) \rightarrow A_{2} \pi\right]=\frac{1}{160}\left(C_{D}^{0}\right)^{2}
$$

$$
\tilde{\Gamma}\left[\rho\left(2^{-}\right) \rightarrow A_{1} \pi\right]=\frac{3}{160}\left(a_{0}^{0}\right)^{2}
$$

$$
\left.\tilde{\Gamma}\left[\eta\left(2^{-+}\right) \underset{N}{\rightarrow} \pi_{N}(980) \pi\right] \frac{1}{160} a_{0}^{0}\right)^{2}
$$

A selection rule, which holds in $S U(6)_{W}\left[\Delta I_{z}=0, \pm 1\right]$ but not in chiral $\mathrm{U}(3) \times \mathrm{U}(3)$, says

$A_{3} \nrightarrow B \pi$

Normally this decay would be allowed in a $d$ wave $(\lambda=1$ only . 


\section{EQUIVALENCES}

a) Rôle of ${ }^{3} \mathrm{p}$

As mentioned in section 3 , the $S U(6)_{\mathrm{W}} \cdot\left[\Delta \mathrm{I}_{\mathrm{z}}=0, \pm 1\right]$ : model is equivalent to the ${ }^{3} P_{0}$ model. What is meant by this equivalence is that there is a one-te-one: cocrespondence between independent parameters: of both models. Strictly speaking one colld argue that the two models cannot be identical since in the $\mathrm{SU}(6)_{\mathrm{W}}\left[\Delta \mathrm{I}_{\mathrm{z}}=0, \pm 1\right]$ model each helicity amplitude for a specific decay channel. $A \rightarrow B+\pi$ is proportional to $m_{A}^{2}-m_{B}^{2}$, because of PCAC, while this factor is not present in the usual formulation of the ${ }^{3} P_{0}$ mojel. However, since the ${ }^{3} \mathrm{P}_{0}$ model is essentially a phenomenological prescription to break $S U(6)$ w, strong symetry for vertices one can always include any function of the external masses in its definition.

The proof of the equivalence between the two models in the above sense is straightforward and not very illuminating. Let us consider the decay amplitudes

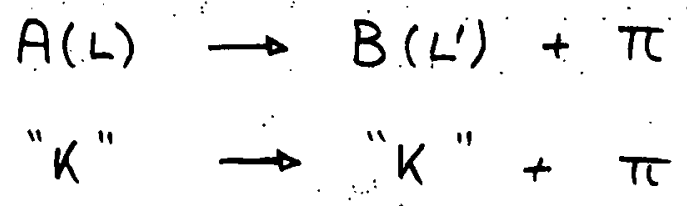

Helicity amplitudes for these decays in the $\operatorname{SJ}(6)_{W} \quad\left[\Delta L_{z}=0, \pm 1\right]$ model can be rewritten as

$$
\begin{aligned}
& g_{\lambda}=\sum_{L_{2}}\left\{\begin{array}{cc|c}
35 & 35 & 35 \\
\left(8,2 w^{8}+1\right) & (8,3) & \underset{\left(8,2 w^{4}+1\right)}{35}
\end{array}\right\}_{\alpha_{1}}\left\{\begin{array}{ll|l}
8 & 8 & 8 \\
B & \pi & A
\end{array}\right\}_{\alpha_{2}} \\
& \left\{a^{L_{z} L_{z}}\left(w^{B} \lambda-L_{z}|0| w^{A} \lambda-L_{z}\right)\left(S^{A} \lambda-L_{z} L_{L} L_{z} \mid J^{A} \lambda\right)\left(S^{B} \lambda-L_{z} L^{\prime} L_{z} \mid J^{B} \lambda\right)\right. \\
& +a^{L_{z}+1 L^{2}}\left(w^{B} \lambda-L_{-}-1|1| w^{A} \lambda-L_{z}\right)\left(S^{A} \lambda-L_{z} L_{z} \mid J^{A} \lambda\right)\left(S^{B} \lambda-L_{-}-1 L^{\prime} L_{q}+1 \mid J^{B} \lambda\right) \\
& \left.\begin{array}{c}
+a^{L-1} L_{2}\left(w^{B} \lambda-L_{z}+1|-1| w^{A} \lambda-L_{z}\right)\left(S^{A} \lambda-L_{z} L_{Z} \mid J^{A} \lambda\right)\left(S^{B} \lambda-L_{z}+\left|L^{1} L_{z}-1\right| J^{B} \lambda\right) \\
\vdots \quad \therefore \quad \cdots
\end{array}\right\}
\end{aligned}
$$

where we have absorbed the factor $\mathrm{m}_{\mathrm{A}}^{2}-\mathrm{m}_{\mathrm{B}}^{2}$ into the reduced matrix elements $a^{L \frac{f}{2} \tau} \frac{1}{z}$ 
In the ${ }^{3} \mathrm{P}_{\mathrm{o}}$ model, on the other hand, the same helicity amplitudes. are parmetrized as follows

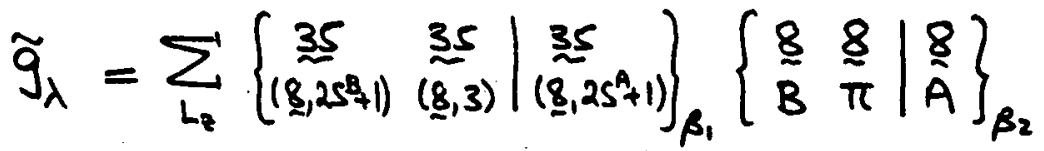

$$
\begin{aligned}
& \left\{b^{L_{z} L_{z}}\left(S^{B} \lambda-L_{z} 10 \mid s^{A} \lambda-L_{2}\right)\left(s^{A} \lambda-L_{2} L L_{z} \mid J^{A} \lambda\right)\left(s^{B} \lambda-L_{z} L^{\prime} L_{2} \mid J^{B} \lambda\right)\right. \\
& +b^{L+14}\left(S^{2} \lambda-L_{z}-111 \mid s^{A} \lambda-L_{z}\right) \cdot\left(S^{A} \lambda-L_{z} L L_{z} \mid J^{A} \lambda\right)\left(S^{B} \lambda-L_{z}-1 L^{\prime} L_{z}+1 \mid J^{B} \lambda\right) \\
& \left.+b^{L-1 L_{z}}\left(S^{B} \lambda-L_{z}+1|-1| S^{A} \lambda-L_{z}\right)\left(S^{A} \lambda-L_{z} L_{z} \mid J^{A} \lambda\right)\left(S^{B} \lambda-L_{z}+1 L^{\prime} L_{z}-1 \mid J^{B} \lambda\right)\right\}_{(4.2)}
\end{aligned}
$$

It is then simple to show that the coefficients of the $a^{I_{z}^{f} L_{z}^{i}}$ It is then simple to show that the coefficients of the a ${ }^{L} \frac{f}{z} L \frac{i}{z}$
reduced matrix elements are proportional to the coefficients of the reduced matrix elements and that the proportionality factor is independent of $I_{g}$. One finds that with the correspondences

or

$$
\begin{aligned}
& a^{L_{z} L_{z}} \rightarrow b^{L_{z}} \\
& a^{L_{z} \pm ! L_{z}} \longrightarrow-\frac{4}{3 \sqrt{2}} b^{L_{z} \pm 1 L_{z}}
\end{aligned}
$$

$$
\begin{aligned}
& a^{L_{2} L_{z}} \rightarrow b^{L_{z}} \\
& a^{L^{4 \pm 1 L_{z}}} \stackrel{\longrightarrow}{\longrightarrow}-\frac{3 \sqrt{2}}{4} b^{4 \pm 1 L_{z}}
\end{aligned}
$$

the helicity amplitudes $g_{\lambda}$ and $\tilde{g}_{\lambda}$ are identical. The correspondence of $\mathrm{Eq} .(4,3)$ is to be used when the coupling

\section{$A$ (quark spintriplet state) $\rightarrow B($ quark spin triplet state $)+\cdots$}

is a type in $\mathrm{SU}(3)$ (i.e., L+L' even) while Eq. (4.4) is to be used when the same coupling is $f$ type in $S U(3)$ (i.e., $L+L$ ! odd). 
This thus completes the formal proof that the $\mathrm{SU}(6)_{\mathrm{W}} \quad\left[\Delta I_{z}=0, \pm 1\right]$. model and the ${ }^{3} \mathrm{p}_{0}$ model are identical up to mass factors.

For the case of baryonic decays, the quark spin and $W$ spin of the baryons are the same. The two pictures are manifestly identical for pionic decays in this case.

b) Rôle of $\stackrel{\ell}{\text { broken }} \mathrm{SU}(6)_{W}$

We shall use here the fact that the ${ }^{3} \mathrm{P}_{\mathrm{O}}$ picture and $\mathrm{SU}(6)_{\mathrm{W}}$ $\left[\Delta I_{z}=0, \pm 1\right]$ have been proven identical for $A(L) \rightarrow B\left(I^{\prime}\right)+\pi$. Then let $\ell^{\prime}$ be the vector difference of $I$ and $L^{\prime}$. One can label amplitudes either by $\ell^{\prime}$ and $\ell$ or by $I_{z}^{i}$ and $I_{z}^{f}$. The relation between the two descriptions is

$$
\begin{aligned}
b_{\ell^{\prime}, l}=\sum_{L_{z}^{\prime} L_{z}^{f}} & \left(L_{L_{z}}^{i} \ell^{\prime} L_{z}^{f}-L_{z}^{i} \mid L^{\prime} L_{z}^{f}\right) \\
& \times\left(\ell^{\prime} L_{z}^{f}-L_{z}^{i} 1 L_{z}^{i}-L_{z}^{f} \mid \ell O\right) b^{L_{z}^{f} L_{z}^{i}}
\end{aligned}
$$

Note that in the second coefficient the fact that the ${ }^{3} \mathrm{P}_{0}$. pair has $\mathrm{I}=1$ has been used. This restriction would not be apparent in the $\mathrm{SU}(6)_{\mathrm{W}}$ $\left[\Delta I_{z}=0, \pm 1\right]$ language.

In narve $\mathrm{SU}(6)_{\mathrm{W}}$ one has only $\mathrm{I}_{\mathrm{z}}^{f}-\mathrm{L}_{\mathrm{z}}^{i}=\Delta \mathrm{I}_{\mathrm{z}}=0$. The amplitudes $a_{\ell^{\prime}}=\ell$ all vanish in this case since the second Clebsch-Gordan coefficient is $(\ell 010 \mid \ell 0)=0$. Moreover, in naive $S U(6)_{W}$, the amplitudes $a_{\ell l}=\ell \pm 1, \ell$ for various $\ell$ have relations among them. These are discarded in $l$ broken. $\mathrm{SU}(6)_{\mathrm{W}}$, and the amplitudes $a_{\ell}=\ell \pm 1, \ell$ treated as free paraneters. On the other hand, one never acquires amplitudes of the form $a_{\ell^{\prime}}=\ell, l^{\bullet}$ Such amplitudes are expected to be present in the. ${ }^{3} \mathrm{p}_{0}$ picture, however, and thus give rise to additional degrees of freedom.

$L_{f}^{f} L_{\frac{i}{z}}$ Exceptions occur in two cases: 1) for $L=L^{\prime}$, one has $b^{L \frac{i}{z} L \frac{f}{z}}=$ $b^{L_{z}^{f} L_{z}^{i}}$. The sum over $L_{z}^{i}$ and $L_{z}^{f}$ in Eq. (4.5) then yields no net contribution to $a_{\ell^{\prime}}=\ell, \ell$ even in $S U(6)_{W} \quad\left[\Delta I_{z}=0, \pm 1\right]$; 2) for $I=0 \quad\left(I^{\prime}=0\right)$ one has only $\ell^{\prime}=I^{\prime} \quad\left(\ell^{\prime}=\mathrm{L}\right)$ and since $\ell=L^{\prime} \pm 1 \quad(\mathrm{I} \pm 1)$ by parity conservation, the amplitude $a_{\ell^{\prime}}=\ell, \ell$ cannot occur.

$$
\text { Thus, for decays } \mathrm{A}(\mathrm{L}) \rightarrow \mathrm{B}\left(\mathrm{L}^{1}\right)+\pi, \quad l \text { broken } \mathrm{SU}(6)_{\mathrm{W}} \text { is always }
$$
a special case of the ${ }^{3} P_{0}$ picture $\left[\right.$ and hence of $S U(6)_{W}\left[\Delta I_{z}=0, \pm 1\right]$. 
It coincides with the latter only when 1) $I=I^{\prime}$ or 2) $I$ or $I^{\prime}$ is zero. The last case applies to all previous phenomenological studies 14)-19).

c) $\mathrm{SU}(3) \times \mathrm{SU}(3)$ subgroups

The predictions of Tables II-V for chiral $\mathrm{SU}(3) \times \mathrm{SU}(3)$ relate only amplitudes of a given helicity to one another. If one decomposes helicity states directly into representations of $S U(6)_{W} \times O(2)_{L_{Z}}$, as we have done, we obtain $\mathrm{SU}(6)_{\mathrm{W}}\left[\Delta \mathrm{I}_{\mathrm{z}}=0, \pm 1\right]$.

There is another way of obtaining the latter symmetry froin the former. In the decays $I=1 \rightarrow I=0+\pi$ (for mesons) if one adjoins the predictions of the coplanar $U(3) \times U(3) 24)$ to those of chiral $U(3) \times U(3)$ one obtains those of $\mathrm{SU}(6)_{\mathrm{W}}\left[\Delta \mathrm{I}_{\mathrm{z}}=0, \pm 1\right]$ 15).

To see this explicitly we compare in Table VII the predictions of the two $U(3) \times U(3)$ symmetries, expressed in terms of relations for helicity amplitudes. The coplanar symmetry links together predictions of chiral symmetry for different helicity amplitudes in just such a way as to give $\mathrm{SU}(6)_{\mathrm{W}} \quad\left[\Delta \mathrm{I}_{\mathrm{z}}=0, \pm 1\right]$.

This relation should be true in general. If we form the algebra containing both the coplanar generators and the chiral generators, it closes upon $S U(6)_{W}$. The selection rules for pionic transitions are, moreover, the same as in $\mathrm{SU}(6)_{\mathrm{W}}\left[\Delta \mathrm{L}_{z}=0, \pm 1\right]$. Since the pion remains bilinear in quarks, it must belong to a 35 , while the $\Delta I_{z}$ properties have already been defined at the lower symmetry level.

If $S U(6)_{W}\left[\Delta I_{z}=0, \pm 1\right]$. turns out not to hold, one may thus be able. to pin the blame either on coplanar or chiral $S U(3) \times S U(3)$, with the other symmetry continuing to hold. Table VII forms the basis for one such exercise [which is not, however, demanded by present data, since both $\mathrm{SU}(3) \times \mathrm{SU}(3)$ 's seem valid].

\section{CONCLUSIONS}

In this paper we have explored some consequences of abstracting certain algebraic properties of the Melosh transformation which relates the generators of $\mathrm{SU}(6)_{\mathrm{W}, \text { strong and }} \mathrm{SU}(6)_{\mathrm{W}}$, currents ${ }^{*}$ When PCAC is used to 
relate pionic decays of resonances to one-particle matrix elements of the axial charge, one obtains a model for the $\mathrm{SU}(6)_{\mathrm{W}}$ properties of these pionic transitions. With this model we have understood why a nalve application of $\mathrm{SU}(6)_{\mathrm{W}}$ to vertices should fail whenever $\Delta I_{\mathrm{z}} \neq 0$ transitions are possible. Furthermore, this PCAC model has been shown equivalent to the ${ }^{3} \mathrm{P}_{0}$ model for decays, which has had some phenomenological success. We note, however, that the PCAC model is only formulated to apply to vertices involving a pseudoscalar meson: the ${ }^{3} P_{0}$ model has also been applied to transitions involving vector mesons ${ }^{32}$. The application of the Nelosh transformation to such decay processes is an interesting open question.

Another phenomenological version of $\mathrm{SU}(6)_{\mathrm{W}}, \quad \ell$ broken $\mathrm{SU}(6)_{\mathrm{W}}$, has been shown to differ in general from $\mathrm{SU}(6)_{\mathrm{W}} \quad\left[\Delta \mathrm{I}_{\mathrm{z}}=0, \pm 1\right]$ although in many simple cases the two prescriptions give the same results.

The application of the Melosh transformation thus provides a theoretical basis for understanding the successes of the ${ }^{3} P_{0}$ model. The implications of such a transformation for photoproduction processes, vector dominance, and for current induced processes in general, are presently under - investigation.

\section{ACKNOWLEDGEMENTS}

We thank S.P. De Alwis for helpful discussions concerning the light-plane formulation of the quark transformation, H.J. Melosh for sending a copy of his thesis, and F.J. Gilman and M. Kugler for useful conversations and correspondence. 


\section{THIS PAGE \\ WAS INTENTIONALLY \\ LEFT BLANK}




\section{TABLE I}

a) Classification of $\left|\mathrm{SS}_{2}\right\rangle$ states of $(\underset{6}{6}, \overline{6})$
according to $\mathrm{U}(3) \times \mathrm{U}(3)$ and $\mathrm{SU}(6)_{\mathrm{W}}$

\begin{tabular}{|c|c|c|}
\cline { 2 - 3 } & $\mathrm{U}(3) \times \mathrm{U}(3)$ & $\mathrm{SU}(6)_{\mathrm{W}}$ \\
\hline $\mathrm{S}=1, \mathrm{~S}_{\mathrm{z}}=1$ & $(\underline{3}, \overline{3})_{1}$ & $(8,3)_{1}$ \\
\hline $\mathrm{S}_{z}=0$ & {$\left[(8,1)_{0}+(1,8)_{0}\right] / \sqrt{ } 2$} & $-(8,1)_{0}$ \\
\hline $\mathrm{S}_{z}=-1$ & $(\underline{3}, \underline{3})_{-1}$ & $-(8,3)_{-1}$ \\
\hline $\mathrm{S}=0, \mathrm{~S}_{z}=0$ & $\left.[8,1)-(1,8)_{0}\right] / \sqrt{ } 2$ & $-(8,3)_{0}$ \\
\hline
\end{tabular}

b) $J^{P C}$ of states considered here

\begin{tabular}{|c|c|c|}
\cline { 2 - 3 } \multicolumn{1}{c|}{} & $\mathrm{S}=1$ & $\mathrm{~S}=0$ \\
\hline $\mathrm{L}=0$ & $1^{--}$ & $0^{-+}$ \\
\hline $\mathrm{L}=1$ & $2^{++}, 1^{++}, 0^{++}$ & $1^{+-}$ \\
\hline $\mathrm{I}=2$ & $3^{--}, 2^{--}, 1^{--}$ & $2^{-+}$ \\
\hline
\end{tabular}

+) $\mathrm{SU}(6)_{\mathrm{W}}$ representations are written as $\left[\alpha_{\mathrm{SU}(3)}, 2 \mathrm{~W}+1 \mathrm{~W}_{\mathrm{Z}}\right.$ 


\section{THIS PAGE \\ WAS INTENTIONALLY \\ LEFT BLANK}




\section{TABLE II}

$$
\mathrm{I}=1 \rightarrow \mathrm{I}=0
$$

\begin{tabular}{|c|c|c|c|}
\hline$\lambda=1$ & Process & $a^{00}=1$ & $a^{01} \lambda=1$ \\
\hline & $2^{++} \rightarrow 1^{--}$ & $+3 / 16$ & $-1 / 4 \sqrt{2}$ \\
\hline & $1^{++} \rightarrow 1^{--}$ & $+3 / 16$ & $1 / 4 \sqrt{2}$ \\
\hline$\lambda=0$ & $1^{+-} \rightarrow 1^{--}$ & 0 & $1 / 4$ \\
\hline$\cdots \cdots$ & $2^{++} \rightarrow 0^{-+}$ & $+\sqrt{3} / 8$ & $-1 / 2 \sqrt{6}$ \\
\hline$\cdots$ & $1^{++} \rightarrow 1^{+-}$ & 0 & $1 / 2 \sqrt{2}$ \\
\hline$\cdots$ & $1^{+-} \rightarrow 1^{--}$ & $+3 / 8 \sqrt{2}$ & 0 \\
\hline $0^{++} \rightarrow 0^{-+}$ & $-(1 / 8) \sqrt{3 / 2}$ & $-1 / 2 \sqrt{3}$ \\
\hline
\end{tabular}

\section{Dictionary}

$$
\begin{aligned}
& \mathrm{SU}(6)_{W}\left[\Delta I_{z}=0, \pm 1\right]: a_{\lambda}^{I_{f} I_{i}}=a^{I_{f} I_{i}} \text { for all } \lambda \\
& \ell \text { broken } S U(6)_{W}: \text { equivalent to } \\
& \quad S U(6)_{W}\left[\Delta I_{z}=0, \pm 1\right] \\
& a_{S} \equiv a^{00}+4 \sqrt{2} a^{01 / 3} \quad(l=0) \\
& a_{D} \equiv a^{00}-2 \sqrt{2} a^{01 / 3} \quad(l=2)
\end{aligned}
$$$$
\text { "Narve" } \mathrm{SU}(6)_{\mathrm{W}} \quad: a^{01}=0, \quad a_{\mathrm{S}}=a_{D}
$$ 


\section{THIS PAGE \\ WAS INTENTIONALLY \\ LEFT BLANK}




\section{TABLE III}

$\mathrm{I}=2 \rightarrow \mathrm{I}=0$

\begin{tabular}{|l|c|c|c|}
\hline$\lambda=1$ & Process & $a_{\lambda=1}^{00}$ & $a^{01} \lambda=1$ \\
\hline & $3^{--\rightarrow 1^{--}}$ & $-1 / 2 \sqrt{10}$ & $+(1 / 4) \sqrt{3 / 5}$ \\
\hline & $2^{--} \rightarrow 1^{--}$ & $-1 / 4 \sqrt{2}$ & $-\sqrt{3 / 16}$ \\
& $2^{-+} \rightarrow 1^{--}$ & 0 & $-3 / 8 \sqrt{2}$ \\
\hline$\lambda=0$ & $1^{--} \rightarrow 1^{--}$ & $-1 / 4 \sqrt{10}$ & $-(3 / 16) \sqrt{3 / 5}$ \\
& & 00 & $a^{01} \lambda=0$ \\
\hline & $3^{--} \rightarrow 0^{-+}$ & $-(1 / 4) \sqrt{3 / 5}$ & $+3 / 4 \sqrt{10}$ \\
\hline & $2^{--} \rightarrow 1^{--}$ & 0 & $-3 / 8$ \\
\hline & $2^{-+} \rightarrow 1^{--}$ & $-1 / 4$ & 0 \\
\hline & $1^{--} \rightarrow 0^{-+}$ & $+1 / 2 \sqrt{10}$ & $+(3 / 4) \sqrt{3 / 20}$ \\
\hline
\end{tabular}

\section{Dictionary}

$\mathrm{SU}(6)_{\mathrm{W}}\left[\Delta I_{z}=0, \pm 1\right]: a_{\lambda}^{\mathrm{I}_{f} I_{i}}=a^{I_{f} I_{i}}$ for all $\lambda$

$\ell$ broken $S U(\sigma)_{W}$ : equivalent to

$$
\mathrm{SU}(6)_{\mathrm{W}} \quad\left[\Delta \mathrm{I}_{\mathrm{z}}=0, \pm 1\right]
$$

$a_{P} \equiv a^{00}+(3 / 2) \sqrt{3 / 2} a^{01} \quad(l=1)$

$\mathrm{a}_{\mathrm{F}} \equiv \mathrm{a}^{00}-\sqrt{3 / 2} \mathrm{a}^{01} \quad(l=3)$

"Naive" $\mathrm{sU}(6)_{\mathrm{W}} \quad: \quad \mathrm{a}^{01}=0, \quad \mathrm{a}_{\mathrm{P}}=a_{\mathrm{F}}$ 


\section{THIS PAGE \\ WAS INTENTIONALLY \\ LEFT BLANK}


TABLE IV

$I=1 \rightarrow I=1$

\begin{tabular}{|c|c|c|c|c|}
\hline \multirow[t]{2}{*}{$\boldsymbol{\lambda}=2$} & Process & $a^{00} \lambda=2$ & $a^{11} \lambda=2$ & $a^{10} \lambda=2$ \\
\hline & $2^{++} \rightarrow 2^{++}$ & 0 & $-1 / 4$ & 0 \\
\hline \multirow[t]{10}{*}{$\lambda=1$} & & $a^{00} \lambda=1$ & $a^{11} \lambda=1$ & $a^{10} \lambda=1$ \\
\hline & $2^{++} \rightarrow 2^{++}$ & $-1 / 8$ & $-\mu / 2$ & $-3 / 8 \sqrt{2}$ \\
\hline & $2^{++} \rightarrow 1^{++}$ & $-1 / 8$ & $+M / 2$ & 0 \\
\hline & $2^{++} \rightarrow 1^{+-}$ & 0 & $-1 / 4 \sqrt{2}$ & $+3 / 16$ \\
\hline & $1^{++} \rightarrow 2^{++}$ & $-1 / 8$ & $+\mu / 2$ & 0 \\
\hline & $1^{++} \rightarrow 1^{++}$ & $-1 / 8$ & $-M / 2$ & $+3 / 8 \sqrt{2}$ \\
\hline & $1^{++} \rightarrow 1^{+-}$ & 0 & $+1 / 4 \sqrt{2}$ & $+3 / 16$ \\
\hline & $1^{+-} \rightarrow 2^{++}$ & 0 & $-1 / 4 \sqrt{2}$ & $+3 / 16$ \\
\hline & $1^{+-} \rightarrow 1^{++}$ & 0 & $+1 / 4 \sqrt{2}$ & $+3 / 16$ \\
\hline & $1^{+-} \rightarrow 1^{+-}$ & 0 & $-\mu$ & 0 \\
\hline \multirow{9}{*}{$\lambda=0$} & & $a^{00} \lambda=0$ & $a^{11} \lambda=0$ & $a^{10} \lambda=0$ \\
\hline & $2^{++} \rightarrow 1^{++}$ & 0 & $-1 / 4 \sqrt{3}$ & $+(1 / 4) \sqrt{3 / 2}$ \\
\hline & $2^{+\cdot+} \rightarrow 1^{+-}$ & $-1 / 2 \sqrt{6}$ & 0 & $-\sqrt{3} / 8$ \\
\hline & $1^{++} \rightarrow 2^{++}$ & 0 & $-1 / 4 \sqrt{3}$ & $+(1 / 4) \sqrt{3 / 2}$ \\
\hline & $1^{++} \rightarrow 0^{++}$ & 0 & $-1 / 2 \sqrt{6}$ & $-\sqrt{3} / 8$ \\
\hline & $1^{+-} \rightarrow 2^{++}$ & $-1 / 2 \sqrt{6}$ & 0 & $-\sqrt{3 / 8}$ \\
\hline & $1^{+-} \rightarrow 0^{++}$ & $+1 / 4 \sqrt{3}$ & 0 & $-(1 / 4) \sqrt{3 / 2}$ \\
\hline & $0^{++} \rightarrow 1^{++}$ & 0 & $-1 / 2 \sqrt{6}$ & $-\sqrt{3} / 8$ \\
\hline & $0^{++} \rightarrow 1^{+-}$ & $+1 / 4 \sqrt{3}$ & 0 & $-(1 / 4) \sqrt{3 / 2}$ \\
\hline
\end{tabular}




\section{Dictionary, Table IV}

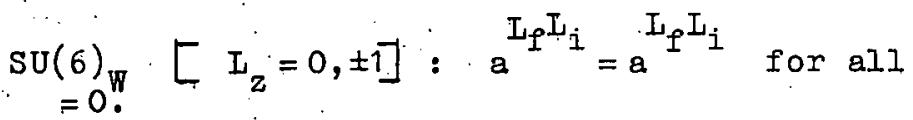

$$
\begin{aligned}
& \dot{\text { broken }} \mathrm{SU}(\sigma)_{\mathrm{W}} \text { : equivalent to } \\
& \mathrm{SU}(6)_{\mathrm{W}}:\left[\Delta I_{z}=0, \pm 1\right] \\
& a_{p}^{0} \equiv a^{00}-(3 / \sqrt{2}) a^{10} \text {. two independent } \\
& a_{p}^{1} \equiv a^{1 \cdot 1}+(3 / 2 \sqrt{2}) a^{10} \quad l=1 \text { combinations } \\
& \mathrm{a}_{\mathrm{F}} \equiv \mathrm{a}^{00}-\mathrm{a}^{11}+(3 / \sqrt{2}) \mathrm{a}^{10} \quad(l=3) \\
& \text { "NaIve" } \mathrm{SU}(6)_{\mathrm{W}}: \quad \mathrm{a}^{10}=0 ; \quad \mathrm{a}_{\mathrm{F}}=a_{\mathrm{P}}^{0}-\mathrm{a}_{\mathrm{P}}^{1}
\end{aligned}
$$

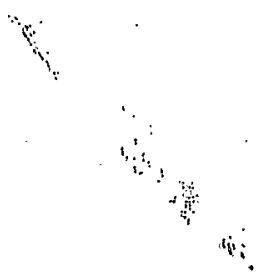


TABLE V

$\mathrm{I}=2 \rightarrow \mathrm{I}=1$

\begin{tabular}{|c|c|c|c|c|c|c|}
\hline \multirow[t]{4}{*}{$\lambda=2$} & Process & $a_{\lambda}^{00}=2$ & $a^{11} \lambda=2$ & $a^{10} \lambda=2$ & ${ }^{a} \lambda=2$ & $a_{\lambda}^{12}=2$ \\
\hline & $3^{--} \rightarrow 2^{++}$ & 0 & $+\sqrt{3} / 8$ & 0 & 0 & $-1 / 4 \sqrt{3}$ \\
\hline & $2^{--} \rightarrow 2^{++}$ & 0 & $+(1 / 8) \sqrt{3 / 2}$ & 0 & 0 & $+1 / 2 \sqrt{6}$ \\
\hline & $2^{-+} \rightarrow 2^{++}$ & 0 & 0 & 0 & 0 & $+1 / 4$ \\
\hline \multirow[t]{13}{*}{$\lambda=1$} & & $a_{\lambda=1}^{\infty O}$ & $a^{11}=1$ & $a_{\lambda}^{10} \lambda=1$ & ${ }^{a} \lambda=1$ & $a_{\lambda=1}^{12}$ \\
\hline & $3^{--} \rightarrow 2^{++}$ & $+3 / 8 \sqrt{10}$ & $-\mu \sqrt{4 / 15}$ & $+1 / 4 \sqrt{5}$ & $-1 / 2 \sqrt{15}$ & $-1 / 4 \sqrt{30}$ \\
\hline & $3^{--} \rightarrow 1^{++}$ & $+3 / 8 \sqrt{10}$ & $+\mu \sqrt{4 / 15}$ & $-1 / 4 \sqrt{5}$ & $-1 / 2 \sqrt{15}$ & $+1 / 4 \sqrt{30}$ \\
\hline & $3^{--} \rightarrow 1^{+-}$ & 0 & $+1 / 4 \sqrt{3 / 5}$ & $-1 / 2 \sqrt{10}$ & 0 & $-1 / 4 \sqrt{15}$ \\
\hline & $2^{--} \rightarrow 2^{++}$ & $+3 / 16 \sqrt{2}$ & $+\mu / \sqrt{12}$ & $+1 / 8$ & $+1 / 8 \sqrt{3}$ & $+1 / 4 \sqrt{6}$ \\
\hline & $2^{--} \rightarrow 1^{++}$ & $+3 / 16 \sqrt{2}$ & $-\mu / \sqrt{12}$ & $-1 / 8$ & $+1 / 8 \sqrt{3}$ & $-1 / 4 \sqrt{6}$ \\
\hline & $2^{--} \rightarrow 1^{+-}$ & 0 & $-\sqrt{3} / 16$ & $-1 / 4 \sqrt{2}$ & 0 & $+1 / 4 \sqrt{3}$ \\
\hline & $2^{-+} \rightarrow 2^{++}$ & 0 & $+3 / 16$ & 0 & $+1 / 4 \sqrt{2}$ & 0 \\
\hline & $2^{-+} \rightarrow 1^{++}$ & 0 & $-3 / 16$ & 0 & $+1 / 4 \sqrt{2}$ & 0 \\
\hline & $2^{-+} \rightarrow 1^{+-}$ & 0 & $-\mu$ & 0 & 0 & 0 \\
\hline & $1^{--} \rightarrow 2^{++}$ & $+3 / 16 \sqrt{10}$ & $+\mu \sqrt{3 / 20}$ & $+1 / 8 \sqrt{5}$ & $+(1 / 8) \sqrt{3 / 5}$ & $-(1 / 4) \sqrt{3} / 10$ \\
\hline & $1^{--} \rightarrow 1^{++}$ & $+3 / 16 \sqrt{10}$ & $-\mu \sqrt{3 / 20}$ & $-1 / 8 \sqrt{5}$ & $+(1 / 8) \sqrt{3 / 5}$ & $+(1 / 4) \sqrt{3 / 10}$ \\
\hline & $1^{--} \rightarrow 1^{+-}$ & 0 & $-(3 / 16) \sqrt{3 / 5}$ & $-1 / 4 \sqrt{10}$ & 0 & $-(1 / 4) \sqrt{3 / 5}$ \\
\hline \multirow[t]{9}{*}{$\lambda=0$} & & $a_{\lambda}^{00}=0$ & $a_{\lambda=0}^{11}$ & $a_{\lambda=0}^{10}$ & $a_{\lambda=0}^{01}$ & $a_{\lambda=0}^{12}$ \\
\hline & $3^{--} \rightarrow 1^{++}$ & 0 & $+3 / 8 \sqrt{5}$ & $-(1 / 2) \sqrt{3 / 10}$ & 0 & 0 \\
\hline & $3^{--} \rightarrow 1^{+-}$ & $+(3 / 8) \sqrt{3 / 10}$ & 0 & 0 & $-1 / 2 \sqrt{5}$ & 0 \\
\hline & $2^{--} \rightarrow 2^{++}$ & 0 & $+(1 / 8) \sqrt{3 / 2}$ & 0 & $+1 / 2 \sqrt{3}$ & 0 \\
\hline & $2^{--} \rightarrow 0^{++}$ & 0 & $+\sqrt{3} / 8$ & .0 & $-1 / 2 \sqrt{6}$ & 0 \\
\hline & $2^{-+} \rightarrow 2^{++}$ & $+\sqrt{ } 3 / 8$ & 0 & $+1 / 2 \sqrt{6}$ & 0 & 0 \\
\hline & $2^{-+} \rightarrow 0^{++}$ & $-(1 / 8) \sqrt{3 / 2}$ & 0 & $+1 / 2 \sqrt{3}$ & 0 & 0 \\
\hline & $1^{--} \rightarrow 1^{++}$ & 0 & $+(3 / 8) \sqrt{3 / 10}$ & $+1 / 2 \sqrt{5}$ & 0 & 0 \\
\hline & $1^{--} \rightarrow 1^{+-}$ & $-3 / 8 \sqrt{5}$ & 0 & 0 & $-(1 / 2) \sqrt{3 / 10}$ & 0 \\
\hline
\end{tabular}




\section{THIS PAGE \\ WAS INTENTIONALLY \\ LEFT BLANK}


Dictionary, Table $V$

$$
\begin{aligned}
& \mathrm{SU}(6)_{\mathrm{W}}\left[\Delta \mathrm{I}_{z}=0, \pm 1\right]: a_{\lambda}^{\mathrm{I}_{f} I_{i}}=a^{\mathrm{L}_{f} \mathrm{~L}_{i}} \text { for all } \lambda ; \mu=0 \\
& \begin{array}{l}
\text { broken } \mathrm{SU}(6) \mathrm{W}_{2}: \\
\mathrm{a}^{10} \sqrt{3}+\mathrm{a}^{01}=\mathrm{a}^{2} \sqrt{2}
\end{array} \\
& a_{S} \equiv a^{00}+a^{11} \sqrt{3}+(8 / 3) \sqrt{2} a^{10}+4 \sqrt{2 / 3} a^{01} \quad(l=0) \\
& a_{D}^{0} \equiv a^{00}-(4 \sqrt{2} / 3) a^{10} \quad \text { (two independent } \\
& a_{D}^{1} \equiv a^{11}-(2 \sqrt{2} / 3) a^{01} \\
& \ell=2 \text { combinations) } \\
& a_{G} \equiv a^{00}-(2 / \sqrt{3}) a^{11}+\sqrt{2} a^{10}-\sqrt{273} a^{01} \quad(l=4) \\
& \text { "Narve" } S U(6)_{W}: a^{01}=a^{10}=a^{12}=0 \text {; } \\
& a_{S}=a_{D}^{0}+a_{D}^{1} \sqrt{3} ; \quad a_{G}=a_{D}^{0}-2 / \sqrt{3} a_{D}^{1}
\end{aligned}
$$




\section{THIS PAGE \\ WAS INTENTIONALLY \\ LEFT BLANK}




\section{$\underline{\text { TABLE VI }}$}

SPECIFIC PARTIAI WIDTH PREDICTIONS

FOR $\quad I=2 \rightarrow I=0+\pi$

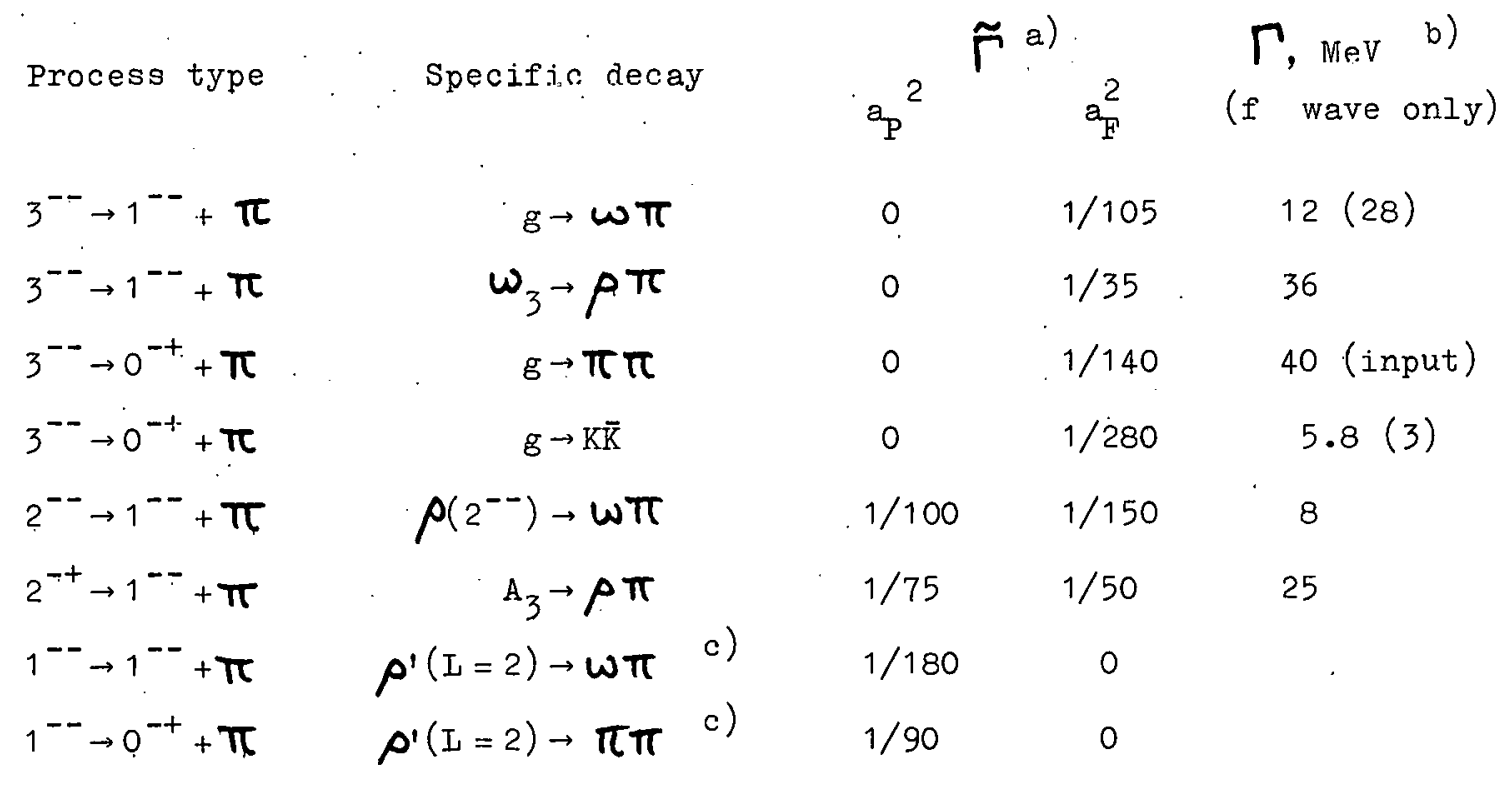

Notes:

a) $\widetilde{\Gamma}_{\ell}=\Gamma_{\ell} \mathrm{M}_{R}^{2}\left(\mathrm{p} / \mathrm{p}_{0}\right)^{-(2 l+1)}$ where $\mathrm{M}_{R}=$ mass of the resonance, $\mathrm{p}$ is the final c.m. three-momentum, and $\mathrm{p}_{\mathrm{o}}$ is some convenient scale factor whose choice of course determines the magnitlide of $a_{\ell}^{2}$.

b) Predictions based on assuming $\Gamma(g \rightarrow \pi \pi)=40 \mathrm{MeV}$ [Ref. 30)]. Experimental numbers, shown in parentheses, are based on Graham and Yoon, [Ref. 31)]. Predictions based on a PCAC kinematic factor are somewhat different and are discussed in the text.

c) Predictions for the $1^{--}$states assume no mixing with the $\mathrm{I}=0$ multiplet ("radial excitation") expected near the same mass. 


\section{THIS PAGE \\ WAS INTENTIONALLY \\ LEFT BLANK}




\section{TABLE VII}

\section{COMPARISON OF $U(3) \times U(3)$ RELAT IONS}

\section{FOR $\quad \mathrm{L}=1 \rightarrow \mathrm{I}=0+\pi \quad \mathrm{DECAYS}$}

\section{COPLANAR}

$$
\begin{aligned}
& g_{1}(2)+g_{1}\left(1^{++}\right)=\sqrt{2} g_{0}\left(1^{+-}\right) \\
& g_{1}(2)=(\sqrt{3} / 2) g_{0}(2) \\
& \sqrt{2} g_{1}\left(1^{+-}\right)=g_{0}\left(1^{++}\right) \\
& \sqrt{2} g_{0}(2)-g_{0}(0)=\sqrt{3} g_{0}\left(1^{+-}\right)
\end{aligned}
$$

\section{CHIRAL}

$$
\begin{aligned}
& g_{1}(2)+\sqrt{2} g_{1}\left(1^{+-}\right)=g_{1}\left(1^{++}\right) \\
& g_{0}(2)+\sqrt{2} g_{0}(0)=-\sqrt{3} g_{0}\left(1^{++}\right) \\
& \sqrt{2} g_{0}(2)-g_{0}(0)=\sqrt{3} g_{0}\left(1^{+-}\right)
\end{aligned}
$$

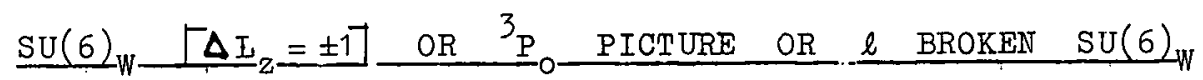

$$
\begin{aligned}
& \mathrm{g}_{1}(2)=(3 / \sqrt{2}) \mathrm{D} \quad \mathrm{g}_{0}\left(1^{++}\right)=\sqrt{2}(\mathrm{~S}-\mathrm{D}) \\
& \mathrm{g}_{0}(2)=\sqrt{6 \mathrm{D}} \\
& g_{1}\left(1^{++}\right)=\sqrt{2}(\mathrm{~S}+\mathrm{D} / 2) \\
& g_{0}(0)=-\sqrt{3} s \\
& g_{0}\left(1^{+-}\right)=S+2 D \\
& g_{1}\left(1^{+-}\right)=S-D
\end{aligned}
$$

\section{Notes}

a) Relation coinmon to coplanar and chiral $U(3) \times U(3)$

b) $D$ and $S$ are conveniently normalized $D$ wave and $S$ wave amplitudes. An equivalent parametrization in terms of $\Delta I_{z}=0, \pm 1$ amplitudes is given in Table II. 


\section{THIS PAGE \\ WAS INTENTIONALLY \\ LEFT BLANK}




\section{$\underline{R E F E R E N C E S}$}

1.) G. Zweig, CERN preprints TH.401 and 412 (1964), unpublished;

M. Gell-Mann, Phys.Letters $\underline{8}, 214$ (1964).

2) M. Gell-Mann, Phys.Rev. 125, 1067 (1962); Physics 1, 63 (1964).

3) J.D. Bjorken and E.A. Paschos, Phys.Rev. 185, 1975 (1969);

H. Fritzsch and M. Gell-Mann, in the Proceedings of the Tel-Aviv Conference on Duality and Symmetry (1971);

J. Kuti and V. Weisskopf, Phys.Rev. D4, 3418 (1971).

4) H.J. Lipkin and S. Meshkov, Phys.Rev.Letters 14, 670 (1965).

5) R. Dashen and M. Gell-Mann, Phys.Rev.Letters 17, 142, 145 (1965); and in Symmetry Principles at High Energy (1966), Coral Gables Conference, W.H. Freeman and Co.

6) S. Fubini and G. Furlan, Physics 1, 229 (1965);

R. Dashen and M. Gell-Mann, Ref. 5);

S. Fubini, G. Segré and J.D. Walecka, Ann.Phys.(N.Y.) 39, 381 (1966).

7) S.I. Adler, Phys.Rev.Letters 14, 1051 (1965);

W. Weisberger, Phys.Rev.Letters 14, 1047 (1965); Phys.Rev. 143, 1302 (1966).

8) See e.g.,

P. Babu, F.J. Gilman and M. Suzuki, Phys.Letters 24B, 65 (1967);

F.J. Gilman and H. Harari, Phys.Rev. 165, 1803 (1968).

9) A recent review of the experimental results is given by

H.W. Kendall in the Proceedings of the International Symposium on Electron and Photon Interactions at High Energies (1971), ed. by: N.B. Mistry (Cornell University Press, Ithaca, N.Y. 1972).

10) See e.g.,

H. Harari, Phys.Rev.Letters 16, 964 (1966); 17, 56 (1966);

I.S. Gerstein and B.W. Lee, Phys.Rev.Letters 16, 1060 (1966);

D. Horn, Phys.Rev.Letters 17, 778 (1966);

F. Buccella, H. Kleinert, C.A. Savoy, E. Celeghini and E. Sorace, Nuovo Cimento 69A, $133(1970)$.

11) H.J.Melosh, Cal.Tech. thesis (1973), unpublished.

12) F.J. Gilman and M. Kugler, Phys.Rev.Letters 30, 518 (1973).

13) A.J.G. Hey and J. Weyers, CERN preprint TH.1614, to be published in Phys.Letters. 
14) I. Micu, Nuclear Phys. B10, 521 (1969).

15) E.W. Colglazier and J.I. Rosner, Nuclear Phys. B27, 349 (1971).

16) W.P. Petersen and J.I. Rosner, Phys.Rev. D6, 820 (1972); D7, 747 (1973).

17) D. Faiman and D.E. Plane, Nuclear Phys. B50, 379 (1972).

18) J.I. Rosner, Appendix to C. Lovelace in Proceedings of the XVI International Conference on High Energy Physics, Vol. 3, ed. J.D. Jackson and A. Roberts (National Accelerator Laboratory, Batavia, 1973). .

19) D. Faiman, J.I. Rosner and J. Weyers, CERN preprint TH.1622 (to be published).

20) H.J. Lipkin and S. Meshkov, Phys.Rev. 143, 1269 (1966).

21) H. Harari, D. Horn, M. Kugler, H.J. Lipkin and S. Meshkov, Phys.Rev. $146,1052(1966)$.

22) See e.g.,

J.E. Mandula, Phys.Rev. 185, 1774 (1969).

23) R. Carlitz and M. Kislinger, Phys.Rev. D2, 336 (1970).

24) J.I. Rosner, Phys.Rev. D6, 1781 (1972).

25) R. Dashen and M. Gell-Mann, Phys.Letters 17, 142, 146 (1965).

26) M. Gell-Mann, Phys.Rev.Letters 14, 77 (1965).

27) C.L. Cook and G. Murtaza, Nuoro Cimento 39, 531 (1965).

28) Particle Data Group, Phys.Letters 39B, 1 (1972).

29) F.J. Gilman, private communication.

30) W. Blum, CERN seminar (February, 1973).

31) R.H. Graham and T.S. Yoon, Phys.Rev. D6, 336 (1972).

32) W.P. Petersen and J.I. Rosner, second of Ref. 16);

J.L. Rosner in Proceedings of the VIII Rencontre Moriond sur les Intéractions Electromagnétiques, to be published (1973). 